\title{
Sensitivity of the attractor of the barotropic ocean model to external influences: approach by unstable periodic orbits
}

\author{
E. Kazantsev \\ INRIA-Lorraine, projet NUMATH, 615, rue du Jardin Botanique, BP101, 54602, Villers-lès-Nancy, Cedex, France
}

Received: 16 October 2000 - Revised: 19 March 2001 - Accepted: 20 March 2001

\begin{abstract}
A description of a deterministic chaotic system in terms of unstable periodic orbits (UPO) is used to develop a method of an a priori estimate of the sensitivity of statistical averages of the solution to small external influences. This method allows us to determine the forcing perturbation which maximizes the norm of the perturbation of a statistical moment of the solution on the attractor. The method was applied to the barotropic ocean model in order to determine the perturbation of the wind field which provides the greatest perturbation of the model's climate. The estimates of perturbations of the model's time mean solution and its mean variance were compared with directly calculated values. The comparison shows that some 20 UPOs is sufficient to realize this approach and to obtain a good accuracy.
\end{abstract}

\section{Introduction}

The problem of global climate change became very important at the end of 20th century. One of the major causes of this importance consists of increasing human activity. Human-induced changes observed on decadal time scales are comparable to natural climate swings. Predicting climate changes has attracted much attention. To be able to better foresee the environmental variations is to be able to adapt our activity to the future.

Understanding and predicting climate changes requires the study of this phenomenon from different points of view. First of all, the study concerns the development of the global monitoring of the climate system, including observations of changes in the climate itself and in factors that force climatic change. The second point consists of the development of climatic models that can test and predict future climatic and environmental responses. The third aspect of this problem is the incorporation of the observational data into the model and its subsiquent validation.

Correspondence to: E. Kazantsev

(Eugene.Kazantsev@iecn.u-nancy.fr)
These three points are actually under intensive study, including model experiments and observational data treatment. Significant progress has been made over the past few decades in providing a wide range of climate predictions and in understanding the key factors of the climate changes, including the human-induced factors.

However, the technique which is actually used in the climate change prediction is essentially an a posteriori technique. One has to first prescribe a factor that may influence the climate (increasing of $\mathrm{CO}_{2}$ in the atmosphere, for example), perform a model integration and obtain the climate response. This approach can neither define the perturbation of the model parameters, which results in the prescribed change of the model climate, nor answer the question "what perturbation of the model parameters would induce the most drastic change in the climatic values of the model?".

To be able to determine the "most dangerous" external influence to the climatic model, one has to solve an inverse problem and use an a priori technique in the prediction of climate changes. One way of developing an a priori technique is based on fluctuation-dissipation relation, obtained by Kraichan (1959) for Hamiltonian systems and used in Branstator and Haupt (1998), Gritsoun and Dymnikov (1999), Gritsoun (2001) for climate models with attractors. This approach is rather simple and can easily been realized, but its precision provides only a qualitative understanding of the problem.

Another way to develop an a priori technique and to obtain a better precision is based on the periodic orbit theory. The notion that unstable periodic orbits (UPOs) may constitute the fundamental building blocks of a chaotic system is argued in many papers (see, for example, Auerbach et al., 1987; Cvitanovic, 1988; Christiansen et al., 1997). Theoretically, the infinite number of UPOs embedded in a chaotic attractor may provide its skeleton. Many dynamical invariants as well as statistical averages of physical measurements can be computed from the infinite set of UPOs.

In practice, we cannot take into account all periodic orbits. However, only a limited number of low-period orbits 
may be sufficient for some purposes. This point of view is argued in Hunt and Ott (1996b), Hunt and Ott (1996a). Numerous recent studies confirm this hypothesis. It is shown that one can approximate attractors of dynamical models using only a set of low-period orbits. The accuracy obtained in the approximation is rather good even when the number of UPOs used is not large. Thus, the attractor of the Lorenz model (Lorenz (1963)), its dimension, Lyapunov exponents and Lyapunov vectors were characterized by the UPO set in Franceschini et al. (1993), Eckhard and Ott (1994) and Trevisan and Pancotti (1998). Attractor dimension and statistical averages of the barotropic ocean model have been approximated by UPOs in Kazantsev (1998).

All of these studies require one to find several UPOs of the model numerically. Sparrow (1982) proposed to use the Newton method to locate unstable periodic orbits on the attractor of the Lorenz model.

However, realization of this method requires $\mathrm{O}\left(N^{3}\right)$ operations per iteration due to the requirement to calculate the matrix of the Newton process and to solve the system of equations with this matrix. Therefore, the method is limited for use only with low-dimensional systems. To find UPOs of high-dimensional systems, one can use the method proposed in Kazantsev (1998). This method requires as many operations per iteration as the model does (usually $\mathrm{O}\left(N^{3 / 2}\right)$ or even $\mathrm{O}(N \ln N)$ ).

The possibility of finding UPOs numerically and using them to approximate the attractor parameters provides a prediction of the attractor variations induced by perturbations of external factors.

In this paper, we describe an algorithm of evaluation of the variations in statistical averages of the model solution caused by perturbations in the right-hand side of the model. These perturbations are suppose to be small and the linear response of the model is considered.

To verify this algorithm and to analyze its properties, we use a very simple ocean model with a strange attractor, namely the barotropic ocean model. This model has been carefully investigated for a last three decades; we know much about its behaviour and attractor.

\section{Estimates of sensitivity of the attractor}

Suppose we have a nonlinear dynamical model written in the form of a system of ordinary differential equations:

$$
\frac{d \omega}{d t}=F(\omega)+f,\left.\quad \omega\right|_{t=0}=\omega^{(0)} .
$$

Here $\omega=\omega(t)$ is an independent variable., which is suppose to be a vector of length $N\left(\omega \in R^{N}\right)$, and $f \in R^{N}$ is a time independent forcing. $F(\omega)$ represents the model dynamics which includes linear and nonlinear terms. This system may be considered the result of the spatial discretisation of some system of partial differential equations.
We suppose that the system (1) possesses a unique solution for any $\omega^{(0)} \in R^{N}$. We write this solution as follows:

$\omega(t)=S\left(\omega^{(0)}, t, f\right)$.

In addition, we presume that the system (1) possesses a chaotic attractor $A$ and a set of unstable periodic orbits which is dense on the attractor.

Let us consider statistical averages of the model solution on the attractor:

$\bar{\omega}=\int_{A} \omega d \mu, \quad \overline{\omega^{2}}=\int_{A} \omega^{2} d \mu, \ldots$

The first expression simply represents the average of the model solution over the attractor; the second expression is the average of the square of each solution's component, etc. The mean variance of each component of the vector $\omega$ can be expressed as

$\sigma=\overline{\omega^{2}}-(\bar{\omega})^{2}$.

The square used in formulas (3) and (4) is not a result of a scalar product. This is a vector with elements equal to the squares of the components.

$\left(\varphi^{2}\right)_{i}=\left(\varphi_{i}\right)^{2}$.

If the system is ergodic, these averages can be approximated by corresponding averages of an infinitely long trajectory:

$\bar{\omega} \sim \lim _{T \rightarrow \infty} \frac{1}{T} \int_{0}^{T} \omega(t) d t, \quad \overline{\omega^{2}} \sim \lim _{T \rightarrow \infty} \frac{1}{T} \int_{0}^{T} \omega^{2}(t) d t$.

If we suppose that the UPO set is dense on the attractor, we can approximate the attractor average by the corresponding averages of UPOs

$\bar{\omega} \sim \lim _{L \rightarrow \infty} \frac{\sum_{k=1}^{L} w_{k} \eta_{k}}{\sum_{k=1}^{L} w_{k}}, \quad \overline{\omega^{2}} \sim \lim _{L \rightarrow \infty} \frac{\sum_{k=1}^{L} w_{k} \zeta_{k}}{\sum_{k=1}^{L} w_{k}}$,

where

$\eta_{k}=\frac{1}{T_{k}} \int_{0}^{T_{k}} \omega_{k}(t) d t, \quad \zeta_{k}=\frac{1}{T_{k}} \int_{0}^{T_{k}} \omega_{k}^{2}(t) d t$

are the average of the $k$ th periodic orbit over its period $T_{k}$ and the average of its square, respectively. Weights $w_{k}$ are written as in Kazantsev (1998). They are proportional to the period of the orbit and inversely proportional to its sum of positive Lyapunov exponents:

$w_{k}=\frac{T_{k}}{\sum_{j} \lambda_{k, j}^{+}}$.

The choice of weights will be discussed later in this paper.

Let us add a small perturbation $\delta f$ to the forcing $f$ in the system (1). We get a perturbed system

$\frac{d \omega^{\prime}}{d t}=F\left(\omega^{\prime}\right)+f+\delta f$. 
Evidently, the system (10) has new averages of the solution and of its square on the attractor:

$\overline{\omega^{\prime}}=\int_{A^{\prime}} \omega^{\prime} d \mu, \quad \overline{\omega^{\prime 2}}=\int_{A^{\prime}}\left(\omega^{\prime}\right)^{2} d \mu$.

The question addressed in this paper is how to find the forcing perturbation $\delta f$ of a given small norm, which maximizes the norm of perturbation of the attractor mean

$\|\delta \bar{\omega}\|=\left\|\overline{\omega^{\prime}}-\bar{\omega}\right\|$,

or the norm of its mean variance

$\|\delta \sigma\|=\left\|\left(\overline{\omega^{\prime 2}}-{\overline{\omega^{\prime}}}^{2}\right)-\left(\overline{\omega^{2}}-\bar{\omega}^{2}\right)\right\|$.

To answer this question, we use the approximation (7) and study the variations in the approximated values of the attractor average and its variance induced by the forcing perturbation.

\subsection{Sensitivity of UPO}

Let us first consider the variation of each particular UPO caused by the change of forcing. Let $\omega^{(0)}$ and $T$ be the initial point and the period of an unstable periodic orbit of the system (1). This means the trajectory of (1), issued from $\omega^{(0)}$ returns back to $\omega^{(0)}$ after time $T$, i.e. $\omega^{(0)}, T$ satisfy the equation

$\omega^{(0)}=S\left(\omega^{(0)}, T, f\right)$.

If we suppose that the forcing perturbation does not produce a bifurcation of the UPO (14), then the initial point and the period of corresponding UPO of the perturbed system (10) must satisfy

$\omega^{\prime(0)}=S\left(\omega^{\prime(0)}, T^{\prime}, f^{\prime}\right)$.

Thus, if $\omega^{\prime(0)}, T^{\prime}$ are the initial point and the period of the periodic orbit of the perturbed system, variations of $\delta \omega^{(0)}=$ $\omega^{\prime(0)}-\omega^{(0)}$ and $\delta T=T^{\prime}-T$ must satisfy the equation

$$
\begin{aligned}
\delta \omega^{(0)}= & S\left(\omega^{\prime(0)}, T^{\prime}, f^{\prime}\right)-S\left(\omega^{(0)}, T, f\right) \\
= & \left.\frac{\partial S}{\partial \omega^{(0)}}\right|_{\omega^{(0)}, T, f} \delta \omega^{(0)}+\left.\frac{\partial S}{\partial T}\right|_{\omega^{(0)}, T, f} \delta T \\
& +\left.\frac{\partial S}{\partial f}\right|_{\omega^{(0)}, T, f} \delta f+o(\|\delta f\|)
\end{aligned}
$$

If the norm of the forcing perturbation is supposed to be small, we may consider only the linear part of (16). We obtain

$$
\begin{aligned}
\left(\left.\frac{\partial S}{\partial \omega^{(0)}}\right|_{\omega^{(0)}, T, f}-I\right) \delta \omega^{(0)} & +\left.\frac{\partial S}{\partial T}\right|_{\omega^{(0)}, T, f} \delta T \\
& =-\left.\frac{\partial S}{\partial f}\right|_{\omega^{(0)}, T, f} \delta f
\end{aligned}
$$

where $I$ is the identity matrix.

Operators presented in (17) have the following meaning. The first one, $\partial S /\left.\partial \omega^{(0)}\right|_{\omega^{(0)}, T, f}$, describes the relationship between the variations in the final point of the orbit and its initial point. This is the matrix of the tangent linear model linearized around UPO and integrated on the period. To simplify notations, we shall denote this operator by $R$

$R=\left.\frac{\partial S}{\partial \omega^{(0)}}\right|_{\omega^{(0)}, T, f}$

The second term in the expression (17) represents the derivative of the solution with respect to time. Using Eqs. (1) and (2), we can write

$\left.\frac{\partial S}{\partial T}\right|_{\omega^{(0)}, T, f}=\left.\frac{d \omega}{d t}\right|_{t=T}=F(\omega(T))+f=F\left(\omega^{(0)}\right)+f(19)$

since the orbit is periodic, i.e. $\omega(T)=\omega^{(0)}$.

The matrix of the right-hand side of (17) is the most complex. This matrix describes the variations in the final point of the UPO due to forcing variations. Numerical evaluation of matrices $R$ and $\partial S / \partial f$ depends on the time scheme. One example of their calculation for the barotropic ocean model is shown in the Appendix.

The expression (17) is not an invertible relationship between forcing and UPO parameters (initial point and period). The reason for this is the noninvertibility of the matrix $(R-I)$, calculated for a periodic orbit. This can easily be seen from the spectrum of the tangent linear model integrated for one period. The moduli of eigenvalues of the matrix $R$ are wellknown Floquet multipliers of the UPO and one of them must be equal to one. This eigenvalue corresponds to the perturbation of the initial point along the orbit. This perturbation is conserved by the model and its amplification factor is equal to one. When an identity matrix is subtracted from the tangent linear model, the difference possesses a zero in the spectrum.

The same fact can be explained from another point of view. So far, any point from the UPO can be chosen as its initial point; no forcing perturbation is necessary to move $\omega^{(0)}$ along the orbit. Thus, there exists a non-zero perturbation of the initial point which corresponds to zero perturbation of forcing, i.e. the matrix is degenerated.

The simplest way to avoid this problem is to fix one component of the vector $\omega^{(0)}$. This technique is well-known in the search of UPOs by the Newton method, where the same matrix must be inverted. In Sparrow (1982), for example, the $z$ variable of the Lorenz system is fixed to be $z=r-1$.

In general, one can fix any component of $\omega^{(0)}$ whose time derivative, determined by the model, is not zero. Let us suppose that the first component is fixed. Then, the length of the vector $\delta \omega^{(0)}$ becomes $N-1$. However, the value of $\delta T$ is also important in this study. If we replace the first component $\left(\delta \omega^{(0)}\right)_{1}$ by $\delta T$, then we obtain a vector of length $N$ which contains all the necessary variables.

We define vector $\xi$, whose first component is $\delta T$, and all other components are equal to the corresponding components of $\delta \omega^{(0)}$.

$\xi_{1}=\delta T, \quad \xi_{k}=\left(\delta \omega^{(0)}\right)_{k}, \quad k=2, \ldots, N$

and $\left(\delta \omega^{(0)}\right)_{1}=0$ 
In order to conserve the equality (17), the first column of the matrix $(R-I)$ must also be replaced by the vector $\partial S /\left.\partial T\right|_{\omega^{(0)}, T, f}(19)$. We shall call this matrix $D_{\xi}$

$$
D_{\xi}=\left[\begin{array}{cccc}
\frac{\partial S}{\partial T}{ }_{1} & (R-I)_{1,2} & \ldots & (R-I)_{1, N} \\
\frac{\partial S}{\partial T}{ }_{2} & (R-I)_{2,2} & \ldots & (R-I)_{2, N} \\
\ldots \ddot{S}_{N} & \ldots & \ldots & \ldots \\
\frac{\partial T_{N}}{\partial T} & (R-I)_{N, 2} & \ldots & (R-I)_{N, N}
\end{array}\right]
$$

Using these notations we obtain

$D_{\xi} \xi=-D_{f} \delta f$,

where $D_{f}=\partial S /\left.\partial f\right|_{\omega^{(0)}, T, f}$.

If the matrix $D_{\xi}$ is not degenerated, Eq. (22) will be used below to find perturbations of initial conditions and of the period of the orbit from the forcing perturbation. The case $\operatorname{det}\left(D_{\xi}\right)=0$ points out the possible bifurcation of the UPO when infinitesimal forcing perturbation is sufficient for drastic change in the orbit and even for its disappearance.

\subsection{Sensitivity of statistical moments of UPO}

An average of an UPO (8) is written by dropping the index $k$ :

$$
\eta\left(\omega^{(0)}, T, f\right)=\frac{1}{T} \int_{0}^{T} \omega(t) d t=\frac{1}{T} \int_{0}^{T} S\left(\omega^{(0)}, t, f\right) d t .
$$

Variation in the UPO average induced by forcing perturbation $\delta f(10)$ can be expressed as

$$
\begin{aligned}
\delta \eta= & \frac{1}{T^{\prime}} \int_{0}^{T^{\prime}} S\left(\omega^{\prime(0)}, t, f^{\prime}\right) d t-\frac{1}{T} \int_{0}^{T} S\left(\omega^{(0)}, t, f\right) d t \\
= & \frac{\partial \eta}{\partial T} \delta T+\frac{\partial \eta}{\partial \omega^{(0)}} \delta \omega^{(0)}+\frac{\partial \eta}{\partial f} \delta f+o(\|\delta f\|) \\
= & \frac{\delta T}{T}\left(\omega^{(0)}-\eta\right)+\frac{1}{T} \int_{0}^{T}\left(\left.\frac{\partial S}{\partial \omega^{(0)}}\right|_{t} \delta \omega^{(0)}\right) d t \\
& +\frac{1}{T} \int_{0}^{T}\left(\left.\frac{\partial S}{\partial f}\right|_{t} \delta f\right) d t,
\end{aligned}
$$

because

$$
\begin{aligned}
\frac{\partial \eta}{\partial T} & =\frac{\partial\left(\frac{1}{T} \int_{0}^{T} S\left(\omega^{(0)}, t, f\right) d t\right)}{\partial T} \\
& =\frac{T S\left(\omega^{(0)}, T, f\right)-\int_{0}^{T} S\left(\omega^{(0)}, t, f\right) d t}{T^{2}} \\
& =\frac{\omega^{(0)}-\eta}{T} .
\end{aligned}
$$

In a discrete time, integrals are replaced by sums

$$
\begin{aligned}
& \frac{\partial \eta}{\partial \omega^{(0)}}=\frac{1}{M} \sum_{m=1}^{M=T / \tau}\left(\left.\frac{\partial S}{\partial \omega^{(0)}}\right|_{t=m \tau}\right), \\
& \frac{\partial \eta}{\partial f}=\frac{1}{M} \sum_{m=1}^{M=T / \tau}\left(\left.\frac{\partial S}{\partial f}\right|_{m \tau}\right) .
\end{aligned}
$$

We recall that the first component of the vector $\delta \omega^{(0)}$ is fixed to be zero and all other components of this vector are combined with the value of $\delta T$ in the vector $\xi(20)$. In this case, the first column in the matrix $\partial \eta / \partial \omega^{(0)}$ can be replaced by the vector $\partial \eta / \partial T=\left(\omega^{(0)}-\eta\right) / T$, similarly to (21). We shall call this matrix $G_{\xi}$

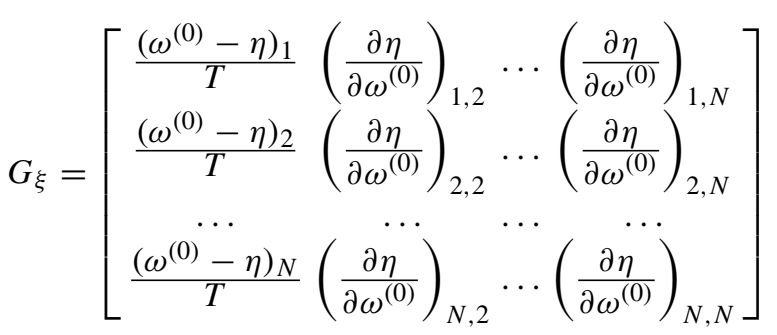

Using these notations and (22) we obtain

$\delta \eta=G_{\xi} \xi+G_{f} \delta f=\left(G_{f}-G_{\xi} D_{\xi}^{-1} D_{f}\right) \delta f$,

where $G_{f}=\partial \eta /\left.\partial f\right|_{\omega^{(0)}, T, f}$ is defined by (26) and $\xi$ is the vector (20). Thus, we obtain a linear relationship between the forcing perturbation and variations of the average of UPO.

Variations of the second moment of UPO can be obtained in a similar way. For each particular UPO

$$
\begin{aligned}
\zeta\left(\omega^{(0)}, T, f\right) & =\frac{1}{T} \int_{0}^{T} \omega^{2}(t) d t \\
& =\frac{1}{T} \int_{0}^{T}\left(S\left(\omega^{(0)}, t, f\right)\right)^{2} d t
\end{aligned}
$$

therefore,

$$
\begin{aligned}
\delta \zeta= & \frac{1}{T^{\prime}} \int_{0}^{T^{\prime}}\left(S\left(\omega^{(0)}, t, f^{\prime}\right)\right)^{2} d t \\
& -\frac{1}{T} \int_{0}^{T}\left(S\left(\omega^{(0)}, t, f\right)\right)^{2} d t \\
= & \frac{\partial \zeta}{\partial T} \delta T+\frac{\partial \zeta}{\partial \omega^{(0)}} \delta \omega^{(0)}+\frac{\partial \zeta}{\partial f} \delta f+o(\|\delta f\|) \\
= & \frac{\delta T}{T}\left(\left(\omega^{(0)}\right)^{2}-\zeta\right) \\
& +\left.\frac{2}{T} \int_{0}^{T} S\left(\omega^{(0)}, t, f\right) \frac{\partial S}{\partial \omega^{(0)}}\right|_{t} \delta \omega^{(0)} d t+ \\
& +\left.\frac{2}{T} \int_{0}^{T} S\left(\omega^{(0)}, t, f\right) \frac{\partial S}{\partial f}\right|_{t} \delta f d t,
\end{aligned}
$$

because

$$
\begin{aligned}
\frac{\partial \zeta}{\partial T} & =\frac{T\left(S\left(\omega^{(0)}, T, f\right)\right)^{2}-\int_{0}^{T}\left(S\left(\omega^{(0)}, t, f\right)\right)^{2} d t}{T^{2}} \\
& =\frac{\left(\omega^{(0)}\right)^{2}-\zeta}{T}
\end{aligned}
$$

Expression (30) is similar to (24). All matrices in this formula have already been calculated. We can only note that the 
product used in this formula is defined by (5). This means

$(\eta \delta \eta)_{i}=\eta_{i} \delta \eta_{i}$ and

$$
\begin{aligned}
& \left(S\left(\omega^{(0)}, t, f\right) \frac{\partial S}{\partial \omega^{(0)}} \delta \omega^{(0)}\right)_{i} \\
& \quad=\left.S_{i}\left(\omega^{(0)}, t, f\right) \sum_{j=1}^{N} \frac{\partial S}{\partial \omega^{(0)}}\right|_{i, j}\left(\delta \omega^{(0)}\right)_{j}
\end{aligned}
$$

Using this notation and Eqs. (28) and (30), we obtain

$\delta \zeta=G_{\xi}^{(2)} \xi+G_{f}^{(2)} \delta f=\left(G_{f}^{(2)}-G_{\xi}^{(2)} D_{\xi}^{-1} D_{f}\right) \delta f$

where

$G_{f}^{(2)}=\left.\frac{2}{T} \int_{0}^{T} S\left(\omega^{(0)}, t, f\right) \frac{\partial S}{\partial f}\right|_{t} d t$,

and

$$
G_{\xi}^{(2)}=\left[\begin{array}{ccc}
\frac{\left(\omega_{0}^{2}-\zeta\right)_{1}}{T}\left(\frac{\partial \zeta}{\partial \omega^{(0)}}\right)_{1,2} & \cdots\left(\frac{\partial \zeta}{\partial \omega^{(0)}}\right)_{1, N} \\
\frac{\left(\omega_{0}^{2}-\zeta\right)_{2}}{T}\left(\frac{\partial \zeta}{\partial \omega^{(0)}}\right)_{2,2} & \cdots\left(\frac{\partial \zeta}{\partial \omega^{(0)}}\right)_{2, N} \\
\cdots & \cdots & \cdots \\
\frac{\left(\omega_{0}^{2}-\zeta\right)_{N}}{T}\left(\frac{\partial \zeta}{\partial \omega^{(0)}}\right)_{N, 2} & \cdots\left(\frac{\partial \zeta}{\partial \omega^{(0)}}\right)_{N, N}
\end{array}\right]
$$

\subsection{Sensitivity of the stability of UPO}

In order to use the approximation of the attractor average (7), we need to calculate the variations of weights (9) used in this formula. These variations can be easily obtained:

$\delta w=\frac{\delta T\left(\sum_{j} \lambda_{j}^{+}\right)-T\left(\sum_{j} \delta \lambda_{j}^{+}\right)}{\left(\sum_{j} \lambda_{j}^{+}\right)^{2}}$

Each Lyapunov exponent of the UPO $\lambda_{j}$ is the ratio of the logarithm of the Floquet multiplier and the period of orbit. Floquet multipliers are obtained as moduli of eigenvalues of the matrix $R(18)$

$\lambda_{j}=\frac{\ln \left|\mu_{j}\right|}{T}$,

hence,

$\delta \lambda_{j}=\frac{1}{T^{2}}\left(\frac{T}{\left|\mu_{j}\right|} \delta\left|\mu_{j}\right|-\delta T \ln \left|\mu_{j}\right|\right)$.

Therefore,

$$
\begin{aligned}
& \delta w= \\
& =\frac{\delta T\left(\sum_{j} \lambda_{j}^{+}\right)-T \sum_{j} \frac{1}{T^{2}}\left(\frac{T}{|\mu|_{j}^{+}} \delta|\mu|_{j}^{+}-\delta T \ln |\mu|_{j}^{+}\right)}{\left(\sum_{j} \lambda_{j}^{+}\right)^{2}} \\
& =\frac{T \delta T\left(\sum_{j} \lambda_{j}^{+}\right)-T \sum_{j} \frac{\delta|\mu|_{j}^{+}}{|\mu|_{j}^{+}}+\delta T \sum_{j} \ln |\mu|_{j}^{+}}{T\left(\sum_{j} \lambda_{j}^{+}\right)^{2}}
\end{aligned}
$$

$$
=\frac{2 \delta T}{\left(\sum_{j} \lambda_{j}^{+}\right)}+\frac{\sum_{j} \frac{\delta|\mu|_{j}^{+}}{|\mu|_{j}^{+}}}{\left(\sum_{j} \lambda_{j}^{+}\right)^{2}} .
$$

The $+\operatorname{sign}$ in $\mu_{j}^{+}$is used to mark Floquet multipliers which are larger than one and which correspond to positive Lyapunov exponents $\lambda_{j}^{+}$.

Using a linear approach, we express variations in the absolute value of $\mu$ in the case when $\mu$ is complex:

$\delta\left|\mu_{j}\right|=\frac{\operatorname{Re}\left(\mu_{j}\right) \operatorname{Re}\left(\delta \mu_{j}\right)+\operatorname{Im}\left(\mu_{j}\right) \operatorname{Im}\left(\delta \mu_{j}\right)}{\left|\mu_{j}\right|}$.

Let us consider variations in $\delta \mu_{j}$ produced by the forcing perturbation.

$R \varphi_{j}=\mu_{j} \varphi_{j}$, where $R=\left.\frac{\partial S}{\partial \omega^{(0)}}\right|_{\omega^{(0)}, T, f}$

Let us suppose that the forcing variation $\delta f$ perturbes the matrix $R$ by $\delta R$. Let us first find the variations in $\mu_{j}$ :

$(R+\delta R)\left(\varphi_{j}+\delta \varphi_{j}\right)=\left(\mu_{j}+\delta \mu_{j}\right)\left(\varphi_{j}+\delta \varphi_{j}\right)$

hence, in the linear framework,

$\left(R-\mu_{j} I\right) \delta \varphi_{j}=-\left(\delta R-\delta \mu_{j} I\right) \varphi_{j}$

Yet $\mu_{j}$ is an eigenvalue of $R$; therefore, the matrix $\left(R-\mu_{j} I\right)$ is degenerated. If we require that $\delta \varphi_{j}$ exists, the right-hand side must be orthogonal to the kernel of adjoint matrix $(R-$ $\left.\mu_{j} I\right)^{*}$, i.e. to the vector $\varphi_{j}^{*}$, such that $\left(R-\mu_{j} I\right)^{*} \varphi_{j}^{*}=0$. In other words, $\varphi_{j}^{*}$ is the eigenvector of $R^{*}$ associated with the complex conjugate eigenvalue $\bar{\mu}_{j}$ :

$R^{*} \varphi_{j}^{*}=\bar{\mu}_{j} \varphi_{j}^{*}$.

The orthogonality condition writes

$\left\langle\left(\delta R-\delta \mu_{j} I\right) \varphi_{j}, \varphi_{j}^{*}\right\rangle=0$,

or

$\left\langle\delta R \varphi_{j}, \varphi_{j}^{*}\right\rangle-\delta \mu_{j}\left\langle\varphi_{j}, \varphi_{j}^{*}\right\rangle=0$.

Let us suppose that all eigenvalues of the matrix are simple. In this case, the scalar product $\left\langle\varphi_{j}, \varphi_{j}^{*}\right\rangle$ is not equal to 0 and we can write

$\delta \mu_{j}=\frac{\left\langle\delta R \varphi_{j}, \varphi_{j}^{*}\right\rangle}{\left\langle\varphi_{j}, \varphi_{j}^{*}\right\rangle}$,

where $\langle.,$.$\rangle is a scalar product in R^{N}$ or in $C^{N}$ when eigenvectors are complex.

In order to evaluate the matrix $\delta R$ for any small forcing perturbation, we write the linear part of the variation

$\delta R=\frac{\partial R}{\partial \omega^{(0)}} \delta \omega^{(0)}+\frac{\partial R}{\partial T} \delta T+\frac{\partial R}{\partial f} \delta f$

Derivatives $\partial R / \partial \omega^{(0)}$ and $\partial R / \partial f$ are three-dimensional tensors. Their calculation requires $N^{4}$ operations per time step. 
However, if we do not need to calculate them explicitly, we can reduce the number of operations. We can note that to calculate $\delta \mu_{j}$ (47), we only need to calculate products

$\frac{\partial R}{\partial \omega^{(0)}} \varphi_{j}$ and $\frac{\partial R}{\partial f} \varphi_{j}$.

These products, which are matrices, require $N^{3}$ operations.

The algorithm of calculation depends on the time discretisation scheme. One example of this algorithm can be seen in the Appendix. The algorithm allows us to calculate two vectors, $d_{\xi}$ and $d_{f}$, as products

$$
\begin{aligned}
\left(d_{\xi}\right)_{k} & =\frac{1}{\left\langle\varphi, \varphi^{*}\right\rangle} \sum_{i, j} \frac{\partial R_{i, j}}{\partial \omega_{k}^{(0)}} \varphi_{j} \varphi_{i}^{*}, \quad \forall k=2,3, \ldots, N \\
\left(d_{\xi}\right)_{1} & =\frac{\left\langle\frac{\partial R}{\partial T} \varphi, \varphi^{*}\right\rangle}{\left\langle\varphi, \varphi^{*}\right\rangle} \text { and } \\
\left(d_{f}\right)_{k} & =\frac{1}{\left\langle\varphi, \varphi^{*}\right\rangle} \sum_{i, j} \frac{\partial R_{i, j}}{\partial f_{k}} \varphi_{j} \varphi_{i}^{*} .
\end{aligned}
$$

Taking into account (47), (48) and (50), we obtain

$$
\begin{aligned}
\delta \mu & =\left\langle d_{\xi}, \xi\right\rangle+\left\langle d_{f}, \delta f\right\rangle \\
& =-\left\langle d_{\xi}, D_{\xi}^{-1} D_{f} \delta f\right\rangle+\left\langle d_{f}, \delta f\right\rangle \\
& =\left\langle d_{f}-D_{f}^{*}\left(D_{\xi}^{-1}\right)^{*} d_{\xi}, \delta f\right\rangle
\end{aligned}
$$

thanks to (22). Using this formula, we can calculate the variations of weights (39)

$$
\begin{aligned}
& \delta w=\frac{2 \delta T}{\left(\sum_{j} \lambda_{j}^{+}\right)} \\
& +\frac{1}{\left(\sum_{j} \lambda_{j}^{+}\right)^{2}} \sum_{j} \frac{\left\langle\left(d_{f}\right)_{j}-D_{f}^{*}\left(D_{\xi}^{-1}\right)^{*}\left(d_{\xi}\right)_{j}, \delta f\right\rangle}{\left|\mu_{j}^{+}\right|}
\end{aligned}
$$

Taking into account that $\delta T$ can be obtained as the first component of the vector $\xi$, we obtain from (22)

$\delta T=-\left(D_{\xi}^{-1} D_{f} \delta f\right)_{1}$.

We can express it as a scalar product with the vector $p$, which is defined as

$$
\begin{aligned}
& p_{1}=1, \quad p_{k}=0, k=2, \ldots N \\
& \delta T=\left\langle-D_{\xi}^{-1} D_{f} \delta f, p\right\rangle=\left\langle-D_{f}^{*}\left(D_{\xi}^{-1}\right)^{*} p, \delta f\right\rangle .
\end{aligned}
$$

In this case,

$$
\begin{aligned}
& \delta w=\frac{1}{\left(\sum_{j} \lambda_{j}^{+}\right)^{2}} \times \\
& {\left[2\left(\sum_{j} \lambda_{j}^{+}\right)\left\langle-D_{f}^{*}\left(D_{\xi}^{-1}\right)^{*} p, \delta f\right\rangle\right.} \\
& \left.\quad+\sum_{j} \frac{\left\langle\left(d_{f}\right)_{j}-D_{f}^{*}\left(D_{\xi}^{-1}\right)^{*}\left(d_{\xi}\right)_{j}, \delta f\right\rangle}{\left|\mu_{j}^{+}\right|}\right] \\
& =\langle h, \delta f\rangle,
\end{aligned}
$$

where

$$
\begin{gathered}
h=\frac{1}{\left(\sum_{j} \lambda_{j}^{+}\right)^{2}}\left[-2\left(\sum_{j} \lambda_{j}^{+}\right) D_{f}^{*}\left(D_{\xi}^{-1}\right)^{*} p\right. \\
\left.+\sum_{j} \frac{\left(d_{f}\right)_{j}-D_{f}^{*}\left(D_{\xi}^{-1}\right)^{*}\left(d_{\xi}\right)_{j}}{\left|\mu_{j}^{+}\right|}\right] .
\end{gathered}
$$

2.4 Approximation of perturbation of the attractor average Using formulas (7), (28) and (57) we obtain

$$
\begin{gathered}
\delta \bar{\omega} \sim \lim _{L \rightarrow \infty} \frac{\sum_{k=1}^{L}\left(\delta w_{k} \eta_{k}+w_{k} \delta \eta_{k}\right)}{\sum_{k=1}^{L} w_{k}} \\
-\frac{\sum_{k=1}^{L} \delta w_{k} \sum_{k=1}^{L} w_{k} \eta_{k}}{\left(\sum_{k=1}^{L} w_{k}\right)^{2}} \\
=\frac{\sum_{k=1}^{L}\left(\delta w_{k} \eta_{k}+w_{k} \delta \eta_{k}\right)-\bar{\omega} \sum_{k=1}^{L} \delta w_{k}}{\sum_{k=1}^{L} w_{k}} \\
=\frac{1}{\sum_{k=1}^{L} w_{k}} \sum_{k=1}^{L}\left[\left\langle h_{k}, \delta f\right\rangle\left(\eta_{k}-\bar{\omega}\right)\right. \\
\left.\quad+w_{k}\left(G_{f}-G_{\xi} D_{\xi}^{-1} D_{f}\right)_{k} \delta f\right] \\
=H^{(1)} \delta f,
\end{gathered}
$$

where matrix $H$ is defined by

$$
\begin{aligned}
H_{i, j}^{(1)}=\frac{1}{\sum_{k=1}^{L} w_{k}} \sum_{k=1}^{L}\left[h_{j}^{(k)}\left(\eta_{k}-\bar{\omega}\right)_{i}\right. \\
\left.+w_{k}\left(G_{f}-G_{\xi} D_{\xi}^{-1} D_{f}\right)_{k}\right] .
\end{aligned}
$$

Perturbation of the mean variance of the solution on the attractor $\sigma=\overline{\omega^{2}}-\bar{\omega}^{2}$ from (3) can be approximated also with the help of the matrix $H^{(2)}$. To develop this matrix, we use expressions (7), (30), and (57)

$$
\begin{aligned}
\delta \sigma \sim \lim _{L \rightarrow \infty} \frac{\sum_{k=1}^{L}\left(\delta w_{k} \zeta_{k}+w_{k} \delta \zeta_{k}\right)}{\sum_{k=1}^{L} w_{k}} & -\frac{\sum_{k=1}^{L} \delta w_{k} \sum_{k=1}^{L} w_{k} \zeta_{k}}{\left(\sum_{k=1}^{L} w_{k}\right)^{2}}-2 \bar{\omega} \delta \bar{\omega} \\
\sim & \frac{\sum_{k=1}^{L}\left(\delta w_{k} \zeta_{k}+w_{k} \delta \zeta_{k}\right)-\overline{\omega^{2}} \sum_{k=1}^{L} \delta w_{k}}{\sum_{k=1}^{L} w_{k}}-2 \bar{\omega} \delta \bar{\omega} \\
= & \frac{1}{\sum_{k=1}^{L} w_{k}} \sum_{k=1}^{L}\left[\left\langle h_{k}, \delta f\right\rangle\left(\zeta_{k}-\overline{\omega^{2}}\right)\right. \\
& \left.+w_{k}\left(G_{f}^{(2)}-G_{\xi}^{(2)} D_{\xi}^{-1} D_{f}\right)_{k} \delta f\right]-2 \bar{\omega} H^{(1)} \delta f \\
= & H^{(2)} \delta f,
\end{aligned}
$$

where $H^{(2)}$ is the matrix defined by

$H_{i, j}^{(2)}=\frac{1}{\sum_{k=1}^{L} w_{k}} \sum_{k=1}^{L}\left[h_{j}^{(k)}\left(\zeta_{k}-\overline{\omega^{2}}\right)_{i}\right.$ 


$$
\left.+w_{k}\left(G_{f}^{(2)}-G_{\xi}^{(2)} D_{\xi}^{-1} D_{f}\right)_{k}\right]-2 \bar{\omega}_{i} H_{i, j}^{(1)} .
$$

Equations (59) and (61) can help us to determine if there exists a forcing perturbation which provides the prescribed perturbation of the average solution or its mean variance. Of course, if the matrix $H^{(1)}$ is not degenerated, any small perturbation of the average solution can be obtained by an appropriate forcing perturbation. Otherwise, one has to analyze the kernel of $\left(H^{(1)}\right)^{*}$. If the prescribed perturbation of the average solution is orthogonal to the kernel of the adjoint matrix, we can solve the system (59) and obtain the necessary forcing perturbation. If the perturbation of the average solution is not orthogonal to the kernel, the system (59) possesses no solution and we can state that there is no forcing perturbation which results in the prescribed perturbation of the average solution.

A similar procedure with the $H^{(2)}$ matrix must be performed if we look for a forcing perturbation resulting in a prescribed perturbation of the mean variance.

\section{Barotropic ocean model in a square}

\subsection{Model and its parameters}

We consider barotropic ocean dynamics, i.e. all the thermodynamic effects are neglected and the vertical structure of the ocean is supposed to be uniform. The equation of dynamics of the wind-driven ocean is written for the barotropic vorticity $\omega$

$$
\frac{\partial \omega}{\partial t}+\mathcal{J}(\psi, \omega+\ell)=\mu \Delta \omega-\sigma \omega+f, \quad \omega=\Delta \psi
$$

We assume the $\beta$-plane approximation for the Coriolis parameter $\ell$, which represents the effect of the Earth's rotation in this equation, i.e. we suppose that this parameter is linear in $y$ coordinate: $\ell=\ell_{0}+\beta y$, where $\ell_{0}$ is the value of the Coriolis parameter at the mid-latitude of the basin. We use very simple basin geometry represented by a square box of side length $L=4000 \mathrm{~km}$. We suppose that this basin is located in the middle of North Atlantic, so we take the value of the Coriolis parameter in the middle of basin to be equal to $\ell_{0}=9.3 \times 10^{-5} \mathrm{~s}^{-1}$, and its meridional gradient $\beta=2 \times 10^{-11}(\mathrm{~ms})^{-1}$.

The source of energy in this equation is presented by the atmospheric wind stress applied to the surface. In this paper, we take a steady zonal wind with a classical two gyre antisymmetric pattern. This is seen as a schematic pattern for the mean curl of the wind stress over the North Atlantic ocean in middle latitudes. Its magnitude is equal to

$f=-\frac{2 \pi \tau_{0}}{\rho H L} \sin \frac{2 \pi y}{L}$

where $\tau_{0}=1.1 \mathrm{dyn} / \mathrm{cm}^{2}$ is the wind tension on the surface, $\rho=1000 \mathrm{~kg} / \mathrm{m}^{3}$ is the density of water. The depth of the active wind driven current $H$ has been chosen to be
$500 \mathrm{~m}$. The dissipation in Eq. (63) is composed by the harmonic lateral friction $\mu \Delta \omega$ and the bottom drag parametrized by $\sigma \omega$. Values of friction coefficients used in this paper are $\mu=1250 \mathrm{~m}^{2} / \mathrm{s}$ and $\sigma=5 \times 10^{-8} \mathrm{~s}^{-1}$.

The equation (63) is subjected to impermeability and freeslip boundary conditions

$$
\left.\psi\right|_{\partial \Omega}=0,\left.\quad \omega\right|_{\partial \Omega}=0 .
$$

In order to look for a weak solution to the problem (63), (65) we perform its variational formulation:

$$
\begin{aligned}
&\left\langle\frac{\partial \omega}{\partial t}, \varphi\right\rangle+\langle\mathcal{J}(\psi, \omega+\beta y), \varphi\rangle \\
&=-\mu\langle\nabla \omega, \nabla \varphi\rangle-\sigma\langle\omega, \varphi\rangle+\langle f, \varphi\rangle, \\
&\langle\omega, \varphi\rangle=-\langle\nabla \psi, \nabla \varphi\rangle .
\end{aligned}
$$

for any function $\varphi(\omega, y) \in H_{0}^{1}(\Omega)$. Here, $H_{0}^{1}(\Omega)$ denotes the linear space of functions such that the square is integrable, as well as the square of their first derivatives. Functions in this space must vanish on the boundary of the domain. Brackets $\langle.$, . $\rangle$ denote the $L_{2}$ scalar product:

$\langle\psi, \varphi\rangle=\iint_{\Omega} \psi \varphi d x d y$

\subsection{Discretisation}

The variational formulation (66), (67) of the problem (63) allows one to look for a solution by the finite element method (FEM). So far, the solution produced by the barotropic model of the North Atlantic typically includes a western boundary layer with intense velocity gradients; the advantage of refining the triangulation along the western boundary of the domain is rather clear. This helps one to keep the quality of explicit eddy resolution of the model, while working with a lower number of grid nodes.

In spite of the fact that the number of operations per time step and grid node is much higher for the FE model, the possibility of considerably reducing the number of grid points diminishs the computational cost of a model run. The possibility of having a better working precision with a lower number of grid points is very valuable in this work due to a high number of operations per point.

The package MODULEF Bernadou (1988) has been used to perform a triangulation of a domain. This package produces quasi-regular triangulation of the domain based on the prescribed grid nodes on its boundary. We require the refining of the triangulation near the western boundary and especially in the middle of the domain, where velocity gradients are extremely sharp.

The domain $\Omega$ is covered by a set of non-intersecting triangles. The set of integration points is defined as the union of vertices and mi-edges of triangles. Finite elements of type $P_{2}$ are used here, i.e. the polynomials of the second degree $p_{i}(x, y)=a_{i} x^{2}+b_{i} x y+c_{i} y^{2}+d_{i} x+e_{i} y+f_{i}$. The $i$ th finite element is taken to be equal to 1 at the i-th integration point and zero at all other points. 


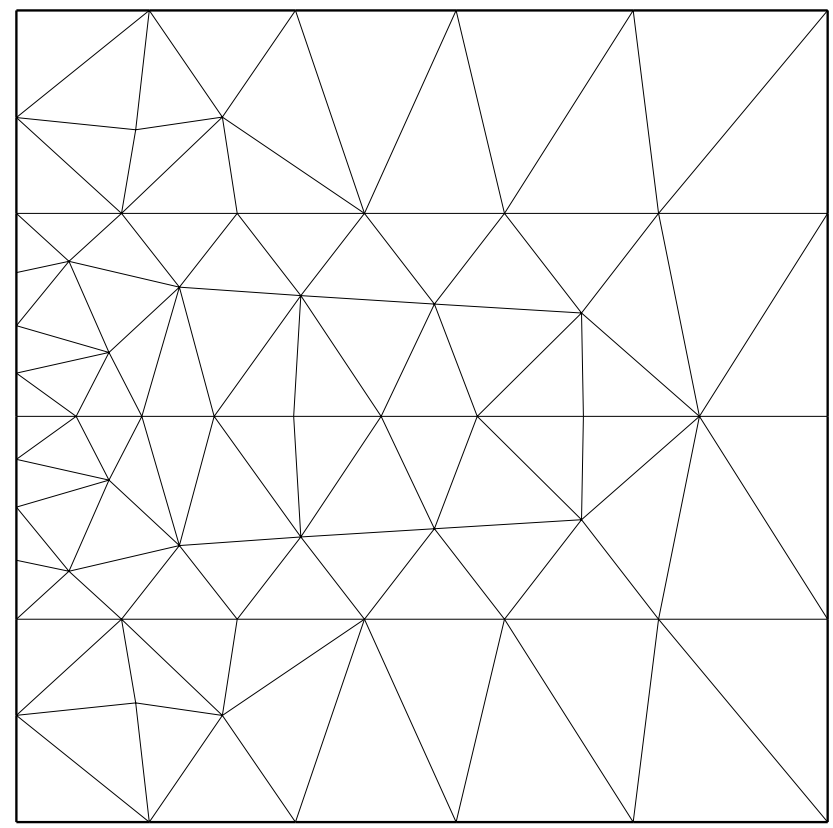

Fig. 1. Triangulation of a unit square. The integration points set is the union of vertices and mi-edges of triangles.

The grid used in this paper is presented in Fig. 1. This triangulation is composed of 92 triangles. The integration points set, which is a union of vertices and mi-edges of triangles, equals 211 nodes. The resolution of the grid varies between $1 / 40$ of the side length (about $100 \mathrm{~km}$ ) near the western boundary, and $1 / 7$ of the side length (about $550 \mathrm{~km}$ ) near the eastern one.

According to the Dirichlet boundary conditions (65), we only consider internal points of the domain: $\left(x_{i}, y_{i}\right) \in \Omega \backslash \partial \Omega$ for $i=1, \ldots, N$., so the functions $\psi, \omega$ are presented as linear combinations

$\psi(x, y, t)=\sum_{i=1}^{N} \psi_{i}(t) p_{i}(x, y)$,
$\omega(x, y, t)=\sum_{i=1}^{N} \omega_{i}(t) p_{i}(x, y)$.

To simplify notations, we define matrices of mass and rigidity as

$$
\begin{aligned}
& \mathcal{M}_{i, j}=\left\langle p_{i}, p_{j}\right\rangle, \\
& \mathcal{C}_{i, j}=\left\langle\nabla p_{i}, \nabla p_{j}\right\rangle
\end{aligned} \quad\left\{\begin{array}{l}
i=1, \ldots, N \\
j=1, \ldots, N
\end{array}\right.
$$

Using these expressions, we can write the discretised system (66):

$$
\begin{aligned}
& \mathcal{M} \frac{\partial \omega}{\partial t}+\mathcal{J}(\psi, \omega+\beta y)=-\mu \mathcal{C} \omega-\sigma \mathcal{M} \omega+\mathcal{M} f \\
& \mathcal{M} \omega=-\mathcal{C} \psi
\end{aligned}
$$

and

$$
\mathcal{J}(\psi, \omega+\beta y)_{j}=\sum_{i} \sum_{m} \psi_{m}\left(\omega_{i}+\beta y_{i}\right)\left\langle\mathcal{J}\left(p_{m}, p_{i}\right), p_{j}\right\rangle
$$

This system has been forwarded in time by the following scheme,

$$
\begin{aligned}
\mathcal{M} & \frac{\omega^{n+1}-\omega^{n-1}}{2 \tau}+\mathcal{J}\left(\psi^{n}, \omega^{n}+\beta y\right) \\
& =-\mu \mathcal{C} \frac{\omega^{n+1}+\omega^{n-1}}{2}-\sigma \mathcal{M} \frac{\omega^{n+1}+\omega^{n-1}}{2}+\mathcal{M} f .
\end{aligned}
$$

The first step is performed by the second order scheme for the nonlinear advection term:

$$
\begin{aligned}
\mathcal{M} & \frac{\omega^{1 / 2}-\omega^{0}}{\tau / 2}+\mathcal{J}\left(\psi^{0}, \omega^{0}+\beta y\right) \\
& =-\mu \mathcal{C} \frac{\omega^{1 / 2}+\omega^{0}}{2}-\sigma \mathcal{M} \frac{\omega^{1 / 2}+\omega^{0}}{2}+\mathcal{M} f \\
\mathcal{M} & \frac{\omega^{1}-\omega^{0}}{\tau}+\mathcal{J}\left(\psi^{1 / 2}, \omega^{1 / 2}+\beta y\right) \\
& =-\mu \mathcal{C} \frac{\omega^{1}+\omega^{0}}{2}-\sigma \mathcal{M} \frac{\omega^{1}+\omega^{0}}{2}+\mathcal{M} f
\end{aligned}
$$

\section{Sensitivity of the barotropic ocean model}

We do not intend to reproduce actual oceanographic data, which would be hopeless in the framework of a barotropic model. This model is used in this paper just as a simple, well studied geophysical model to test the approximation of the attractor by periodic orbits and to test the possibility of explaining the sensitivity of a multi-dimensional chaotic system by means of its unstable periodic orbits.

The purpose of this chapter is threefold:

- to find the external influence $\delta f$ which maximizes the norm of the perturbation of the average of the solution of the barotropic ocean model on its attractor $\|\delta \bar{\omega}\|$ and the mean variance of the solution $\|\delta \sigma\|$,

- to estimate the quantity of UPO necessary to construct matrices $H^{(1)}$ and $H^{(2)}$,

- to compare the perturbation predicted by a linear approach with the reference perturbation produced by this forcing and to verify to what extent the linear approach remains valid. The reference perturbation is calculated from a long trajectory using the formula (6).

In order to study the sensitivity of the model attractor, we formulate first the tangent linear model. This model can be obtained easily as the linearisation of the (63) around its trajectory $S\left(\omega^{(0)}, t, f\right)$ for $t \in[0, T]$ :

$$
\begin{aligned}
\frac{\partial \delta \omega}{\partial t}+ & \mathcal{J}\left(\Delta^{-1} S\left(\omega^{(0)}, t, f\right), \delta \omega\right)+\mathcal{J}\left(\delta \psi, S\left(\omega^{(0)}, t, f\right)+\ell\right) \\
& =\mu \Delta(\delta \omega)-\sigma \delta \omega, \\
\Delta(\delta \psi) & =\delta \omega .
\end{aligned}
$$

This model describes the linear evolution of a small perturbation $\delta \omega$ that has been added to the basic trajectory $S\left(\omega^{(0)}, t, f\right)$. The tangent linear model has been discretised in the same way as the model (63). Finite elements were 
used to perform spatial discretisation and the scheme (70) is applied for time stepping.

$$
\begin{aligned}
\mathcal{M} & \frac{\delta \omega^{n+1}-\delta \omega^{n-1}}{2 \tau}+\mathcal{J}\left(\Delta^{-1} S\left(\omega^{(0)}, t^{n}, f\right), \delta \omega^{n}\right) \\
& +\mathcal{J}\left(\delta \psi^{n}, S\left(\omega^{(0)}, t^{n}, f\right)+\beta y\right) \\
& =-\mu \mathcal{C} \frac{\delta \omega^{n+1}+\delta \omega^{n-1}}{2}-\sigma \mathcal{M} \frac{\delta \omega^{n+1}+\delta \omega^{n-1}}{2} .
\end{aligned}
$$

As seen before, the first step is performed by the scheme for the nonlinear advection term,

$$
\begin{aligned}
& \mathcal{M} \frac{\delta \omega^{1 / 2}-\delta \omega^{0}}{\tau / 2}+\mathcal{J}\left(\Delta^{-1} S\left(\omega^{(0)}, 0, f\right), \delta \omega^{0}\right) \\
&+\mathcal{J}\left(\delta \psi^{0}, S\left(\omega^{(0)}, 0, f\right)+\beta y\right) \\
&=-\mu \mathcal{C} \frac{\delta \omega^{1 / 2}+\delta \omega^{0}}{2}-\sigma \mathcal{M} \frac{\delta \omega^{1 / 2}+\delta \omega^{0}}{2} \\
& \mathcal{M} \frac{\delta \omega^{1}-\delta \omega^{0}}{\tau}+ \mathcal{J}\left(\Delta^{-1} S\left(\omega^{(0)}, \tau / 2, f\right), \delta \omega^{1 / 2}\right) \\
&+\mathcal{J}\left(\delta \psi^{1 / 2}, S\left(\omega^{(0)}, \tau / 2, f\right)+\beta y\right) \\
&=-\mu \mathcal{C} \frac{\delta \omega^{1}+\delta \omega^{0}}{2}-\sigma \mathcal{M} \frac{\delta \omega^{1}+\delta \omega^{0}}{2}
\end{aligned}
$$

Unstable periodic orbits have been found for this model using the method proposed in Kazantsev (1998). For each orbit, we construct matrices $R(18), D_{\xi}, D_{f}(21), G_{\xi}, G_{f}$ (27), $G_{\xi}^{(2)}, G_{f}^{(2)}(35),(34)$, as well as vectors $d_{\xi}, d_{f}(50)$ and $h(58)$. The numerical algorithm of construction of these matrices and vectors is presented in the Appendix.

This allows us to calculate matrices $H^{(1)}(60)$ and $H^{(2)}$ (62), which describe the linear part of relationship the between forcing variation and the average solution on the attractor, and its mean variance.

\subsection{The choice of the weight}

One important question addressed in this paper is the choice of the weight, $w$, used in the approximation of the attractor by UPO set (7). All calculations above have been performed for the weight defined by the formula (9). However, the choice of this weight is absolutely empiric and requires some discussion.

The use of Lyapunov exponents as a measure of instability of UPO is not well justified. A more classical measure used for non-dissipative systems is the Floquet multiplier, $|\mu|=$ $\exp (\lambda T)$. If the system is hyperbolic, then the UPO set is dense in the invariant set; zeta functions and cycle expansion can be used to perform accurate computations. This is much less evident when we work with a dissipative system such as an ocean model.

It has been argued in Eckhard and Grossman (1994) and in Cvitanovic (1995) to use weights proportional to $\left|\operatorname{det}\left[I-R^{\perp}\right]\right|^{-1}$ for hyperbolic systems. Here, the symbol $\perp$ means the projection orthogonal to the direction of the orbit. One can easily see that this weight is based on a Floquet

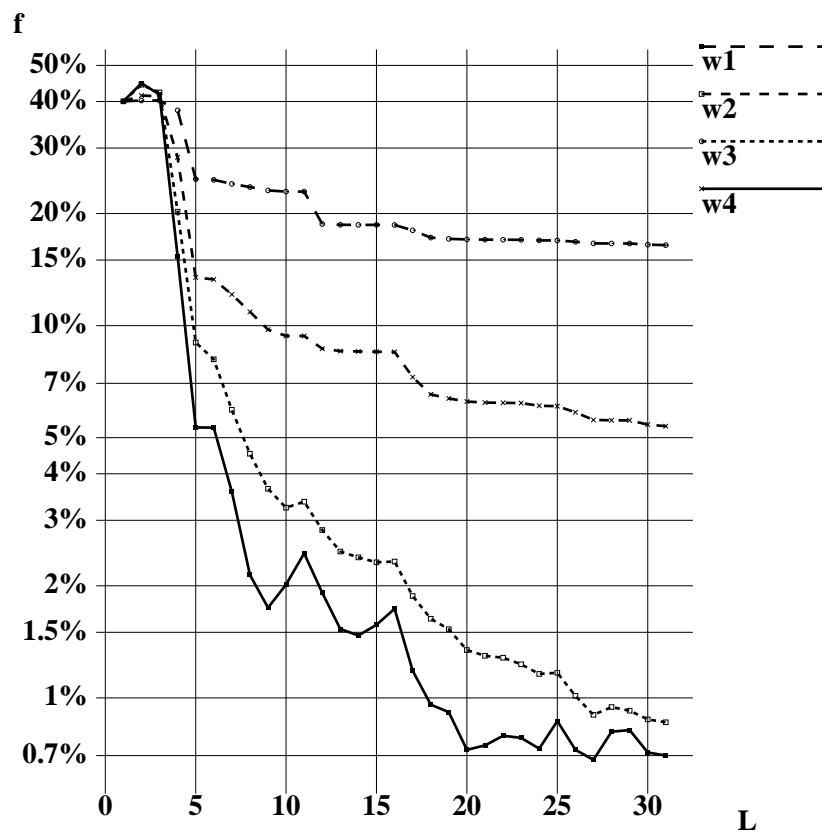

Fig. 2. Difference $\frac{\left\|\bar{\psi}_{\text {approx }}(L)-\bar{\psi}_{\text {direct }}\right\|}{\left\|\bar{\psi}_{\text {direct }}\right\|}$ for different weights $w$.

multiplier:

$$
\begin{aligned}
\left|\operatorname{det}\left[I-R^{\perp}\right]\right|^{-1} & =\left[\prod_{\mu_{k} \neq 1}\left|\left(1-\mu_{k}\right)\right|\right]^{-1} \\
& =\left[\prod_{\lambda_{k} \neq 0}\left|\left(1-e^{\lambda_{k} T}\right)\right|\right]^{-1} .
\end{aligned}
$$

However, statistical averages of UPOs based on cycle expansions developed in the context of known symbolic dynamics of a hyperbolic system may fail in the case of dissipative systems, especially when the symbolic dynamics is not understood. For dissipative systems, it has been proposed in Zoldi and Greenside (1998), Zoldi (1998) to use the escape-time weighting, i.e. weights equal to the inverses sums of positive Lyapunov exponents. The reasoning behind this is rather clear: less unstable orbits must be weighted more heavily.

However, it is also reasonable to suppose that orbits with longer periods must be weighted more heavily. So far, they are longer and should provide greater contribution to the total sum.

In this paper, we compare the approximation of the barotropic model's average solution, approximated using four different weights. The first one is the inverse determinant, $\left|\operatorname{det}\left[I-R^{\perp}\right]\right|^{-1}$, proposed by Eckhard and Grossman (1994). The second one is simply the inverse of the product of all Floquet multipliers greater than one. The third is the escape-time weighting from Zoldi and Greenside (1998), and the fourth is the escape-time multiplied by the period of orbit. 

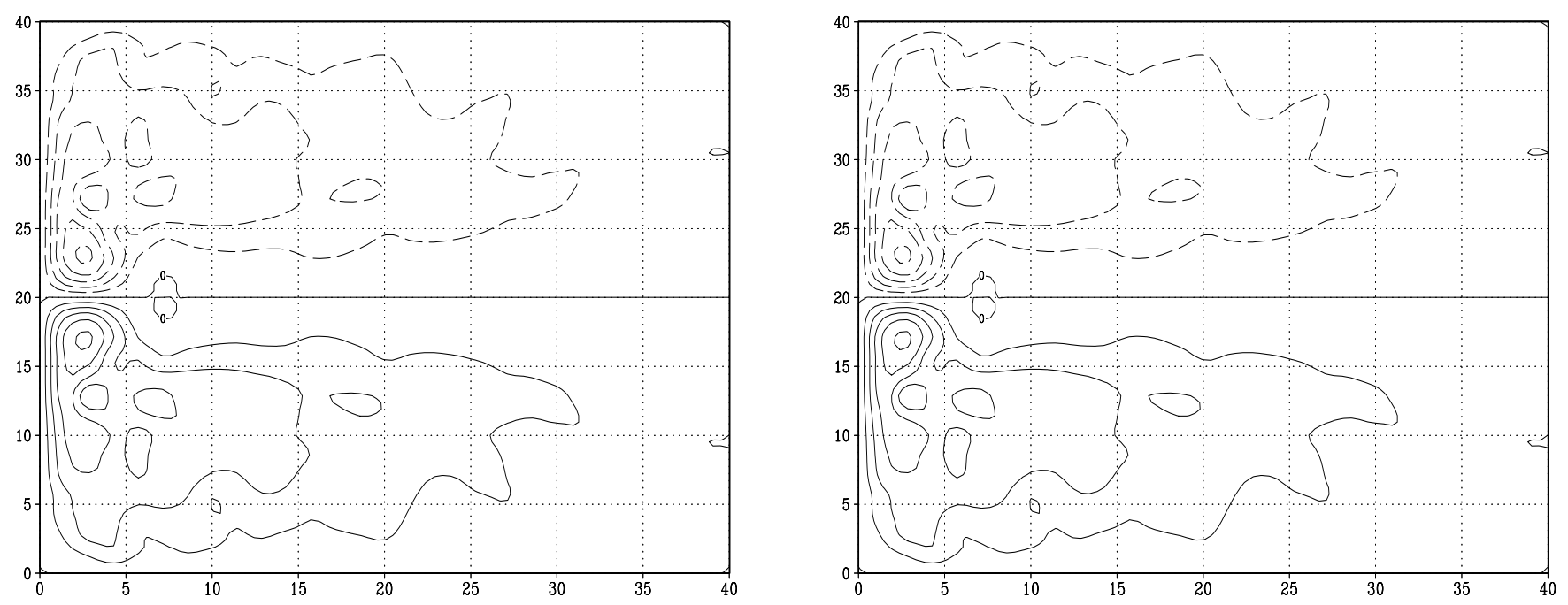

Fig. 3. Average streamfunction calculated by $10^{4}$ years model integration (a) and approximated by the UPO set (b). Contours from -80 to 80 interval $10 \mathrm{~Sv}$.

$$
\begin{aligned}
& w^{(1)}=\left[\prod_{\mu_{k} \neq 1}\left|\left(1-\mu_{k}\right)\right|\right]^{-1} \\
& w^{(2)}=\frac{1}{\exp \left(\sum_{\lambda_{i}>0} \lambda_{i} T\right)} \\
& w^{(3)}=\frac{1}{\sum_{\lambda_{i}>0} \lambda_{i}} \\
& w^{(4)}=\frac{T}{\sum_{\lambda_{i}>0} \lambda_{i}}
\end{aligned}
$$

In order to compare these four weights, we integrate the model for ten thousands years and calculate directly the average solution by (6):

$\overline{\psi_{\text {direct }}}=\frac{1}{10^{4} \text { years }} \int_{0}^{10^{4} \text { years }} \psi(t) d t$,

After that, this average is approximated as a weighted mean of the increasing number of UPOs arranged by period.

$\bar{\psi}_{\text {approx }}(L)=\frac{\sum_{k=1}^{L} w_{k} \eta_{k}}{\sum_{k=1}^{L} w_{k}}, \quad \eta_{k}=\frac{1}{T_{k}} \int_{0}^{T_{k}} \psi_{k}(t) d t$

The relative difference $\left\|\bar{\psi}_{\text {approx }}(L)-\bar{\psi}_{\text {direct }}\right\| /\left\|\bar{\psi}_{\text {direct }}\right\|$ as a function of $L$ is shown in Fig. 2 for different weights $w$. As one can see in Fig. 2, any two weights based on the Floquet multipliers results in a monotonous convergence. But this convergence is much slower than the convergence provided by escape-time weighting. Even 30 orbits are not sufficient to approximate the average with an acceptable accuracy when we use either $w^{(1)}$ or $w^{(2)}$. But $w^{(3)}$ and $w^{(4)}$ provide non-monotonous convergence, which is rather rapid, and we obtain good approximation accuracy. Thirty orbits are necessary to obtain an approximation error less than $1 \%$ using $w^{(3)}$, and only 20 are sufficient for using $w^{(4)}$. This comparison has determined the choice of the weight $w^{(4)}$ as the weight used in this paper.

One can see in Fig. 3 the average streamfunction pattern, calculated by $10^{4}$ years model integration (Fig. 3a) and approximated by the UPO set (Fig. 3b). Thirty UPOs have been used in approximation with weight $w^{(4)}=T / \sum_{\lambda_{i}>0} \lambda_{i}$. The difference between Fig. 3a and Fig. $3 \mathrm{~b}$ is difficult to see. Indeed, as seen in Fig. 2, the relative approximation error is about $0.7 \%$.

We can note here, that Fig. 2 and Fig. 3 also inform us about the quantity of UPO, which is necessary to approximate attractor properties and to obtain a required accuracy. Such a simple experiment shows us whether the number of UPOs we have found is sufficient or not.

\subsection{The "most dangerous" forcing perturbation}

Now we proceed to the sensitivity of the model's attractor. We look for a forcing perturbation to which the attractor average is the most sensible, i.e. we want to find $\delta f$ which maximizes the ratio

$\max \frac{\langle\delta \bar{\omega}, \delta \bar{\omega}\rangle}{\langle\delta f, \delta f\rangle}$

Taking into account (59), we obtain

$$
\begin{aligned}
\max \frac{\langle\delta \bar{\omega}, \delta \bar{\omega}\rangle}{\langle\delta f, \delta f\rangle} & =\max \frac{\left\langle H^{(1)} \delta f, H^{(1)} \delta f\right\rangle}{\langle\delta f, \delta f\rangle} \\
& =\max \frac{\left\langle\left(H^{(1)}\right)^{*} H^{(1)} \delta f, \delta f\right\rangle}{\langle\delta f, \delta f\rangle} \\
& =\max v
\end{aligned}
$$

where $v$ are singular values of $H^{(1)}$

$\left(H^{(1)}\right)^{*} H^{(1)} \delta f=\nu \delta f$ 


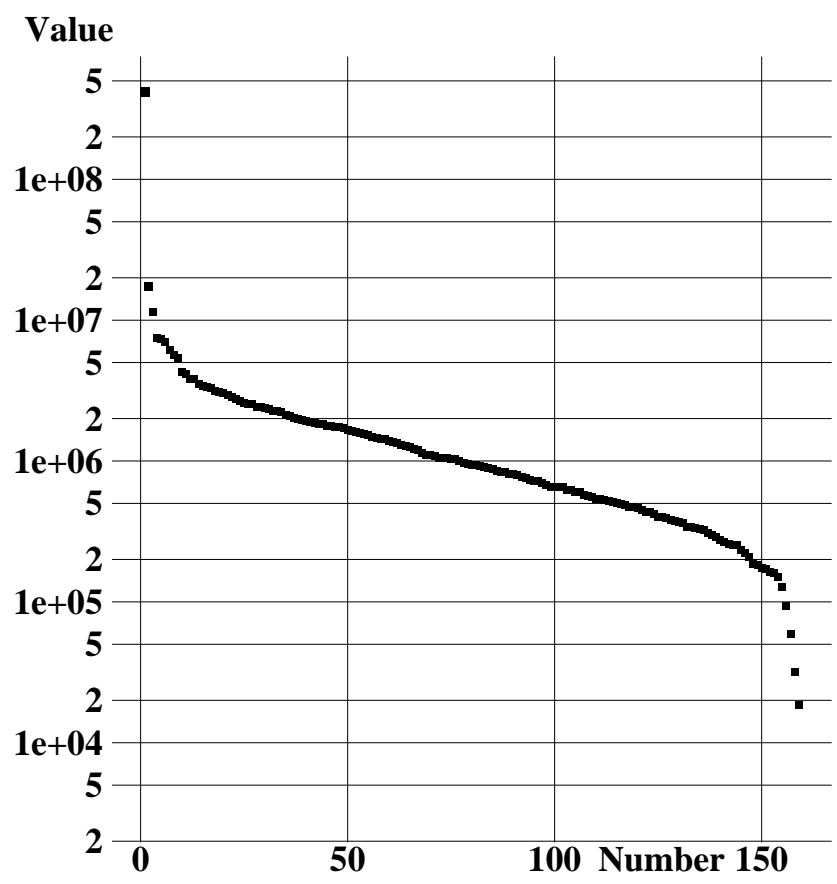

Fig. 4. Singular values of matrices $H^{(1)}$ (a) and $H^{(2)}$ (b).

Thus, the "most dangerous" forcing perturbation can be found as the singular vector of $H^{(1)}$ corresponding to the largest singular value. The value of the ratio $\langle\delta \bar{\omega}, \delta \bar{\omega}\rangle /\langle\delta f, \delta f\rangle$ is expressed by the singular value $v$.

Similarly, the forcing perturbation to which the mean variance of the solution on the attractor is the most sensitive, is expressed as a singular vector of the matrix $H^{(2)}$ corresponding to the largest singular value.

Singular values of $H^{(1)}$ and $H^{(2)}$ are shown in Fig. 4a and Fig. 4b, respectively. Their spectra have been truncated at the 150th value because of a lack of computer accuracy.

One can note the wide range covered by these values, i.e. there exists a forcing perturbation which provokes a drastic changes in the average solution. In addition, the model average exhibits $10^{4}-10^{5}$ times lower sensitivity to some other perturbations of the forcing. One can even see a larger difference in the sensitivity of the mean variance of the solution.

Moreover, there exists a forcing perturbation which is really "the most dangerous" for the attractor. This perturbation corresponds to the first singular vector and it provokes the perturbation of the model average at least 23 times larger than any other $\delta f$ of the same amplitude in the orthogonal space. The first singular value $v$ of the $H^{(1)}$ matrix is equal to $4.15 \times 10^{8}$, the second and the third ones are equal to $1.75 \times 10^{7}$ and $1.14 \times 10^{7}$, respectively.

For the barotropic ocean model, the "most dangerous" $\delta f$ for the average solution is also the "most dangerous" for the mean variance. First singular vectors of $H^{(1)}$ and $H^{(2)}$ coincide. The model's mean variance is also at least 6 times more sensitive to this forcing perturbation than to any other one. The first singular value $v$ of the $H^{(2)}$ matrix is equal to

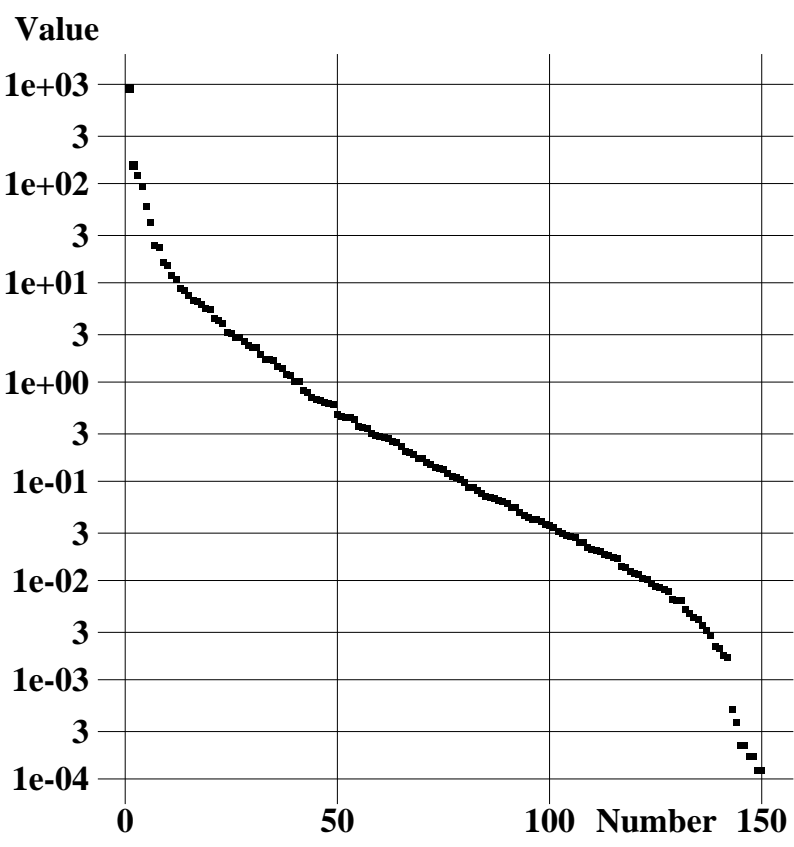

917, the second and the third are 151 and 122 , respectively. This $\delta f$ is the most dangerous for the attractor of the model.

The pattern of this forcing perturbation is shown in Fig. $5 \mathrm{a}$. In spite of the fact that the major model activity takes place near the western boundary, the "most dangerous" $\delta f$ has a maxima near the eastern one. The perturbation which is concentrated in the region of the jet stream of the model corresponds to the third singular vector, which provokes a 25 times lower response of the model average. This forcing perturbation is shown in Fig. $5 \mathrm{~b}$.

The perturbation of the average solution caused by the "most dangerous" $\delta f$ (Fig. 5a) is concentrated in the region of the jet steam near the western boundary. The perturbation of the average streamfunction $\delta \bar{\psi}$ is shown in Fig. 6a, the perturbation of the average vorticity $\delta \bar{\omega}$ in Fig. 7a, and the perturbation of the mean variance $\delta \sigma$ is shown in Fig. $8 \mathrm{a}$. One can point out that only a part of the total basin is shown in these figures, because perturbations are vanishing elsewhere.

This forcing perturbation causes the streamfunction to become asymmetrical. The jet stream in the middle of the basin turns to the north or to the south.

\subsection{Comparison of approximation with direct simulation}

In order to estimate the error of the approximation of the proposed method, we compare this approximation with values calculated directly. Direct calculation has been performed in the following way. We perform first $T=10^{4}$ years integration of the model with original forcing (63) and calculate the 

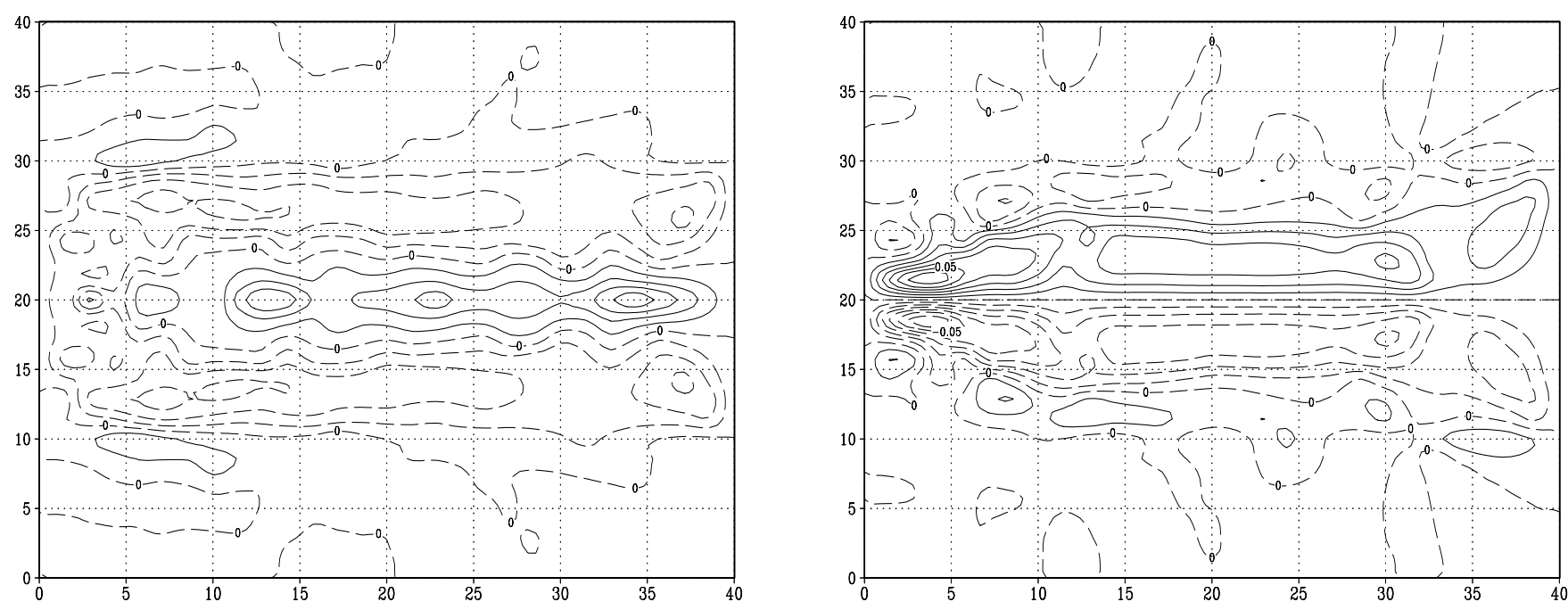

Fig. 5. (a) The "most dangerous" forcing perturbation. (First singular vector of $H^{(1)}$ matrix.) This perturbation results in $\delta\|\bar{\omega}\|=4.15 \times$ $10^{8} \delta\|f\|$. (b) "Less dangerous" forcing perturbation. (Third singular vector of $H^{(1)}$ matrix.) This perturbation results in $\delta\|\bar{\omega}\|=1.14 \times$ $10^{7} \delta\|f\|$.
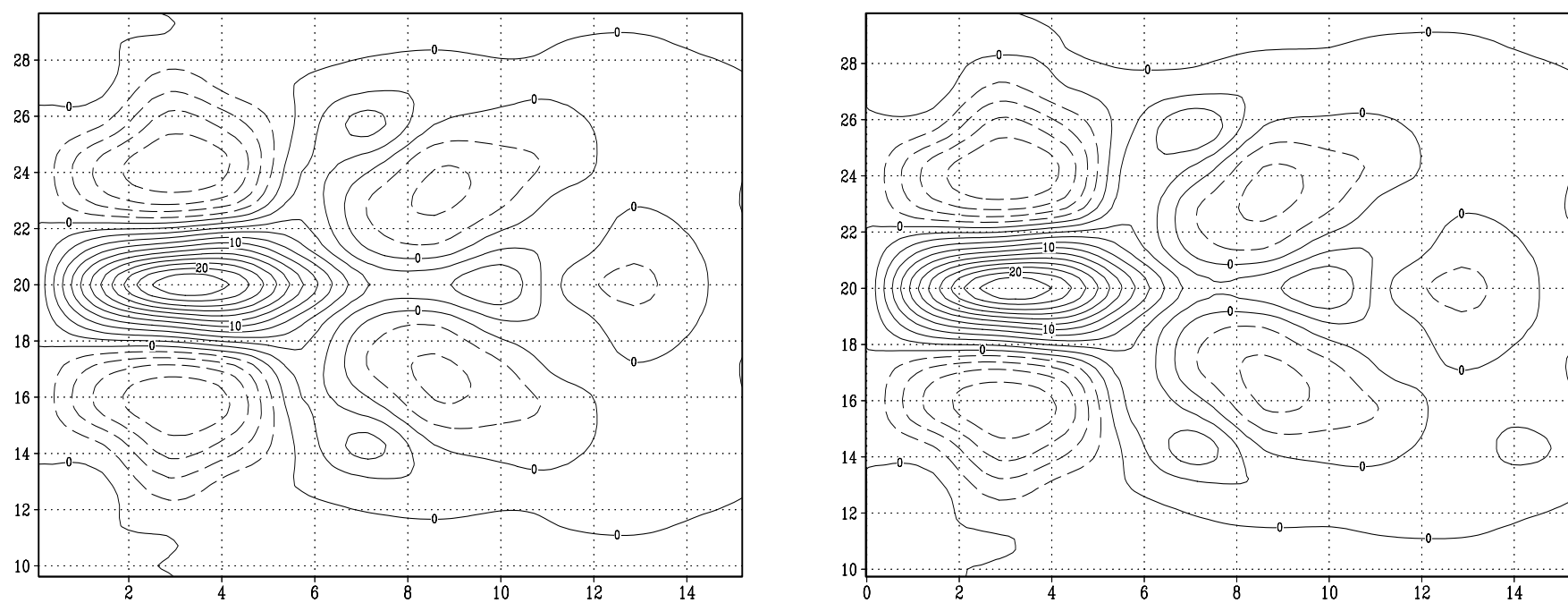

Fig. 6. Perturbation of the streamfunction average approximated by the whole UPO set (a) and calculated by $10^{4}$ years model integration (b). Contours from -10 to 22 interval $2 \mathrm{~Sv}$.

reference averages:

$\bar{\omega}=\frac{1}{T} \int_{0}^{T} \omega(t) d t, \quad \overline{\omega^{2}}=\frac{1}{T} \int_{0}^{T} \omega^{2}(t) d t$.

Second, we perform $T=10^{4}$ years run of the perturbed model with forcing equal to $f+\delta f$ and calculate averages of the perturbed model solution:

$\overline{\omega^{\prime}}=\frac{1}{T} \int_{0}^{T} \omega^{\prime}(t) d t, \quad \overline{\left(\omega^{\prime}\right)^{2}}=\frac{1}{T} \int_{0}^{T}\left(\omega^{\prime}\right)^{2}(t) d t$,

The forcing perturbation used in this run is proportional to the first singular vector of the $H^{(1)}$ matrix in Fig. 5a. The norm of the perturbation is chosen to be $2 \%$ of the original forcing;

$\|\delta f\|=0.02\|f\|$

We calculate the differences between averages of the perturbed model's solution and averages obtained in the reference run. These differences are calculated for the streamfunction $\psi$, vorticity $\omega$ and mean variance of the vorticity $\sigma$.

$\delta \bar{\omega}_{\text {direct }}=\bar{\omega}^{\prime}-\bar{\omega}$

$\delta \bar{\psi}_{\text {direct }}=\bar{\psi}^{\prime}-\bar{\psi}=\Delta^{-1}\left(\bar{\omega}^{\prime}-\bar{\omega}\right)$

$\delta \sigma_{\text {direct }}=\left(\overline{\omega^{\prime 2}}-{\overline{\omega^{\prime}}}^{2}\right)-\left(\overline{\omega^{2}}-\bar{\omega}^{2}\right)$

These values are shown in Fig. 6b, Fig. 7b, and Fig. 8b respectively. They are to be compared with the values ap- 

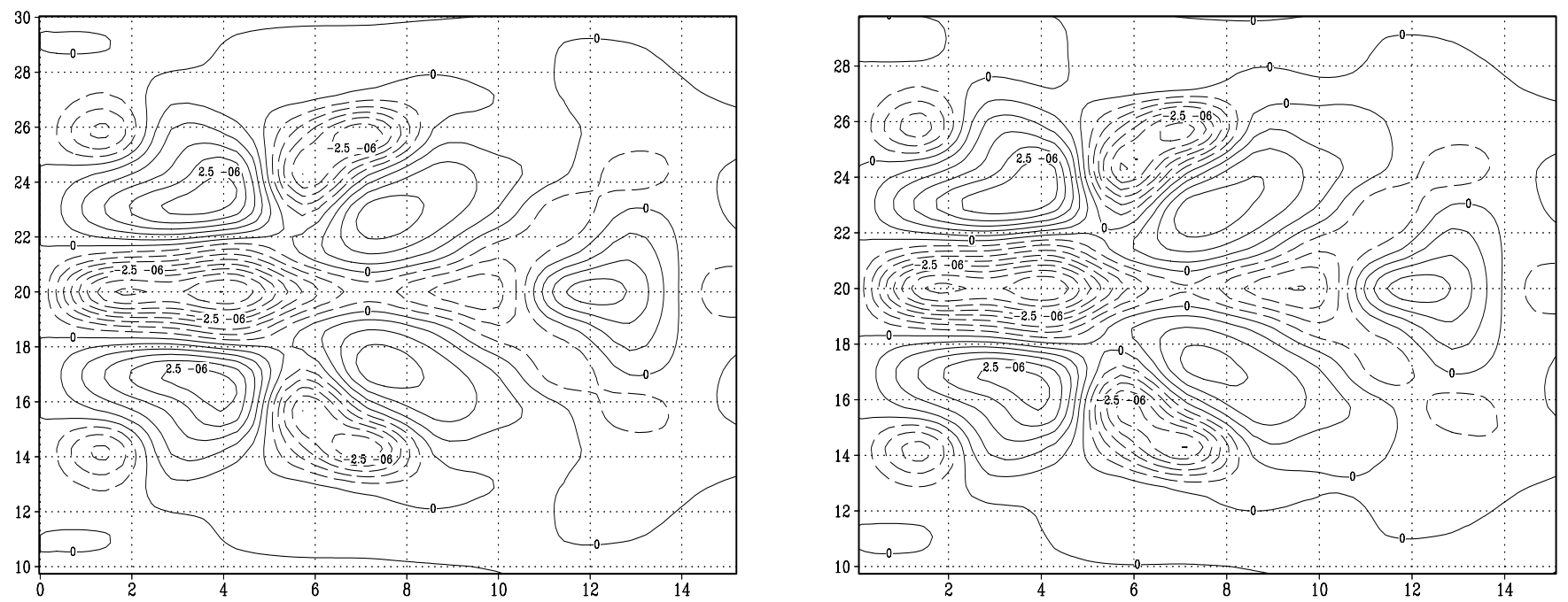

Fig. 7. Perturbation of the vorticity average approximated by the UPO set (a) and calculated by $10^{4}$ years model integration (b). Contours from $-5 \times 10^{-6}$ to $3 \times 10^{-6}$ interval $5 \times 10^{-7} \mathrm{~s}^{-1}$.
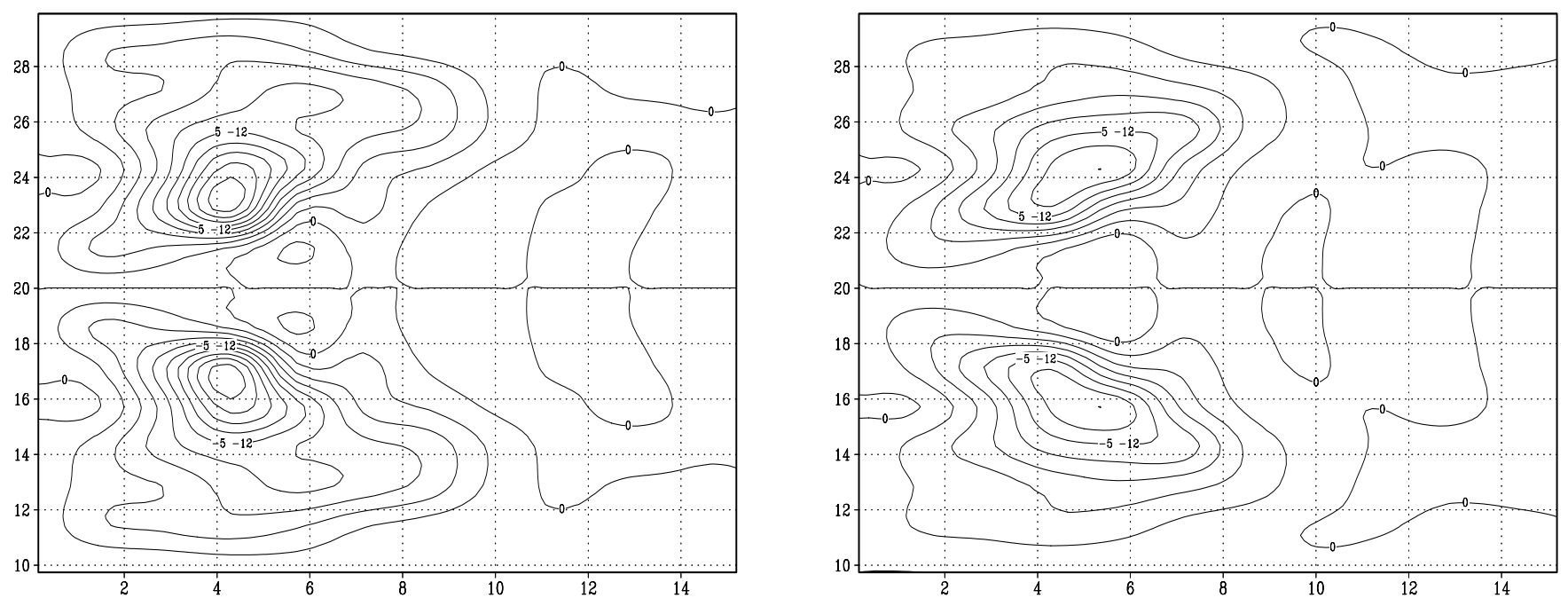

Fig. 8. Perturbation of the mean variance of vorticity approximated by the UPO set (contours from $-9 \times 10^{-12}$ to $9 \times 10^{-12}$ interval $1 \times 10^{-12} \mathrm{~s}^{-2}$ ) (a) and calculated by $10^{4}$ years model integration (contours from $-8 \times 10^{-12}$ to $8 \times 10^{-12}$ interval $1 \times 10^{-12} \mathrm{~s}^{-2}$ ) $(\mathbf{b})$.

proximated by the UPO set, shown in part $A$ of the same pictures. The approximation has been calculated as

$\delta \bar{\omega}_{\text {approx }}(L)=H^{(1)}(L) \delta f$

$\delta \bar{\psi}_{\text {approx }}(L)=\Delta^{-1} H^{(1)}(L) \delta f$

$\delta \sigma_{\text {approx }}(L)=H^{(2)}(L) \delta f$

where $H^{(1)}(L), H^{(2)}(L)$ are matrices computed by (60), (62), using $L$ periodic orbits. All available UPOs were used in the approximations shown in Fig. 6a, Fig. 7a, and Fig. 8a.

As one can see in Fig. 6, Fig. 7, and Fig. 8, there is only a slight difference in the approximation of the mean variance of the vorticity, while patterns of the streamfunction and vorticity seem to be identical to each other. This fact shows that the approximation is rather accurate, especially for averages.
The same comparison can be performed for another forcing perturbation: the third singular vector of the $H^{(1)}$ matrix, in Fig. 5b, for example. This forcing results in the acceleration of the jet stream in the middle of the basin. The jet becomes longer. The perturbation of the streamfunction caused by this forcing is shown in Fig. 9 and it is approximated by the UPO set in Fig. 9a and calculated directly by (86) in Fig. $9 b$.

Now, we will say a few words about the precision of the reconstruction of the attractor perturbation and about the sensitivity of the final result to the number of UPOs used in the reconstruction. As we have seen in Fig. 2, about 20 UPOs are necessary to reconstruct the attractor averages with a precision less than $1 \%$. This precision has been obtained using the weight $w^{(4)}=T / \sum_{\lambda_{i}>0} \lambda_{i}$. We use the same weight in the approximation of the attractor perturbation. 

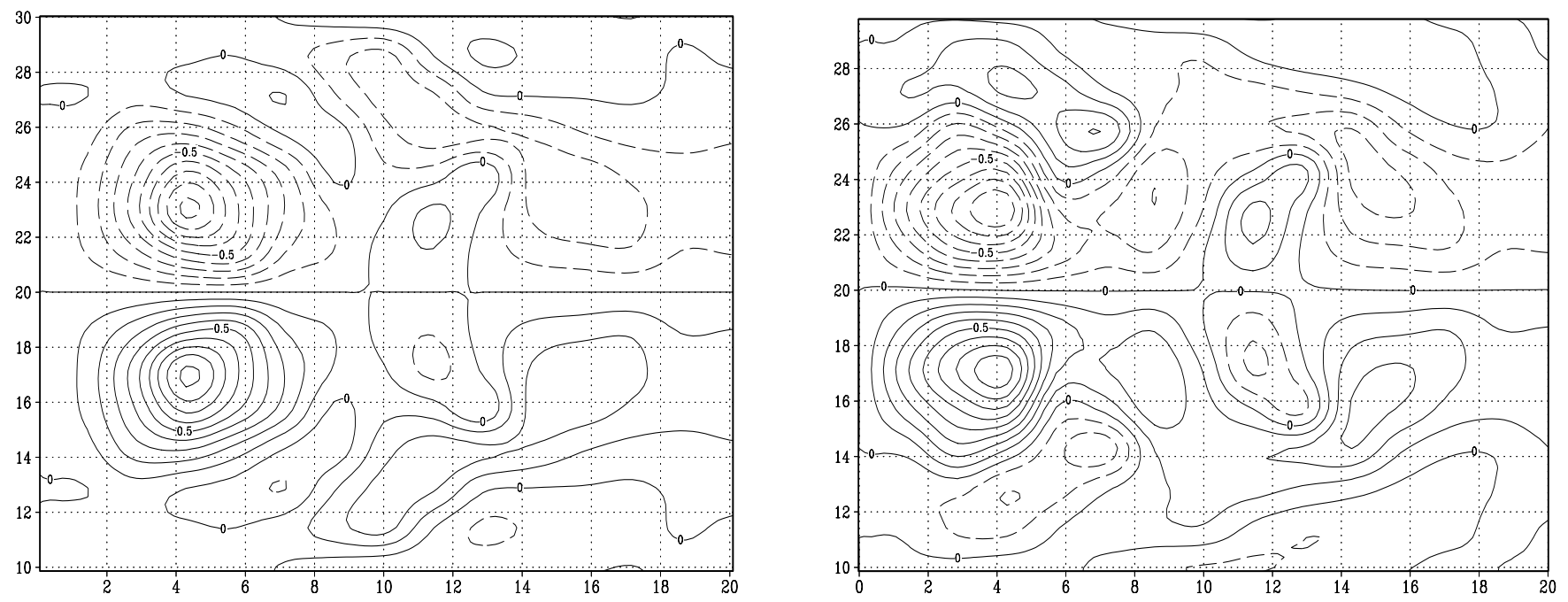

Fig. 9. Perturbation of the streamfunction average approximated by the UPO set (a) and calculated by $10^{4}$ years model integration (b). Contours from -1 to 1 interval $0.1 \mathrm{~Sv}$.
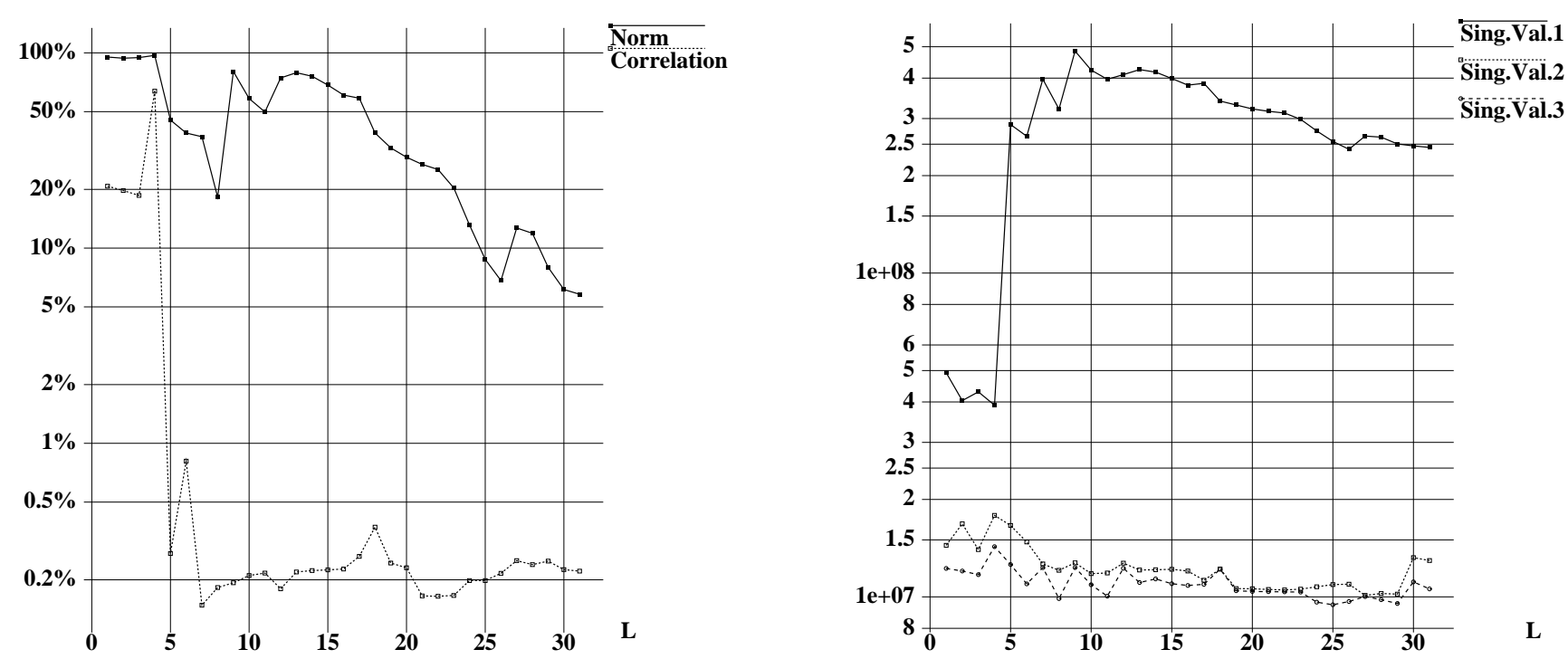

Fig. 10. (a) Values of $1-\alpha(L)$ and $\rho(L)$ for different number of UPOs' $L$. (b) Three largest singular values of $H^{(1)}(L)$ for different number of UPOs' $L$.

To estimate precision we compare the perturbation calculated directly, $\delta \bar{\omega}_{\text {direct }}(85)$, with the reconstructed one, $\delta \bar{\omega}_{\text {approx }}(L)(87)$, obtained using the increasing number $L$ of UPOs arranged by period.

We estimate separately the precision of the reconstruction of the perturbation's pattern and its amplitude. The reconstruction of the pattern is evaluated by the correlation coefficient

$\alpha(L)=\frac{\left\langle\delta \bar{\omega}_{\text {direct }}, \delta \bar{\omega}_{\text {approx }}(L)\right\rangle}{\left\|\delta \bar{\omega}_{\text {direct }}\right\|\left\|\delta \bar{\omega}_{\text {approx }}(L)\right\|}$

where the scalar product is defined by (67). If the correlation coefficient is close to one, thus indicates a good reconstruction of the pattern.
The accuracy of the reconstruction of the amplitude is evaluated by the relative difference in the norm

$\rho(L)=\frac{\left\|\delta \bar{\omega}_{\text {direct }}-\delta \bar{\omega}_{\text {approx }}(L)\right\|}{\left\|\delta \bar{\omega}_{\text {direct }}\right\|}$.

These two values, $\alpha(L)$ and $\rho(L)$, are shown in Fig. 10a for an increasing number of UPOs' $L$ used in reconstruction.

One can see that the pattern of the perturbation is very well reconstructed, even using 5 orbits only with low periods. The correlation coefficient (88) rapidly reaches the value of $99.8 \%$ and an additional increasing of the number of UPOs does not improve it. This fact can be explained by the insufficient accuracy in evaluation of $\delta \bar{\omega}_{\text {direct }}$, due to the finite integration time (10 000 years). Indeed, when we look at Fig. 

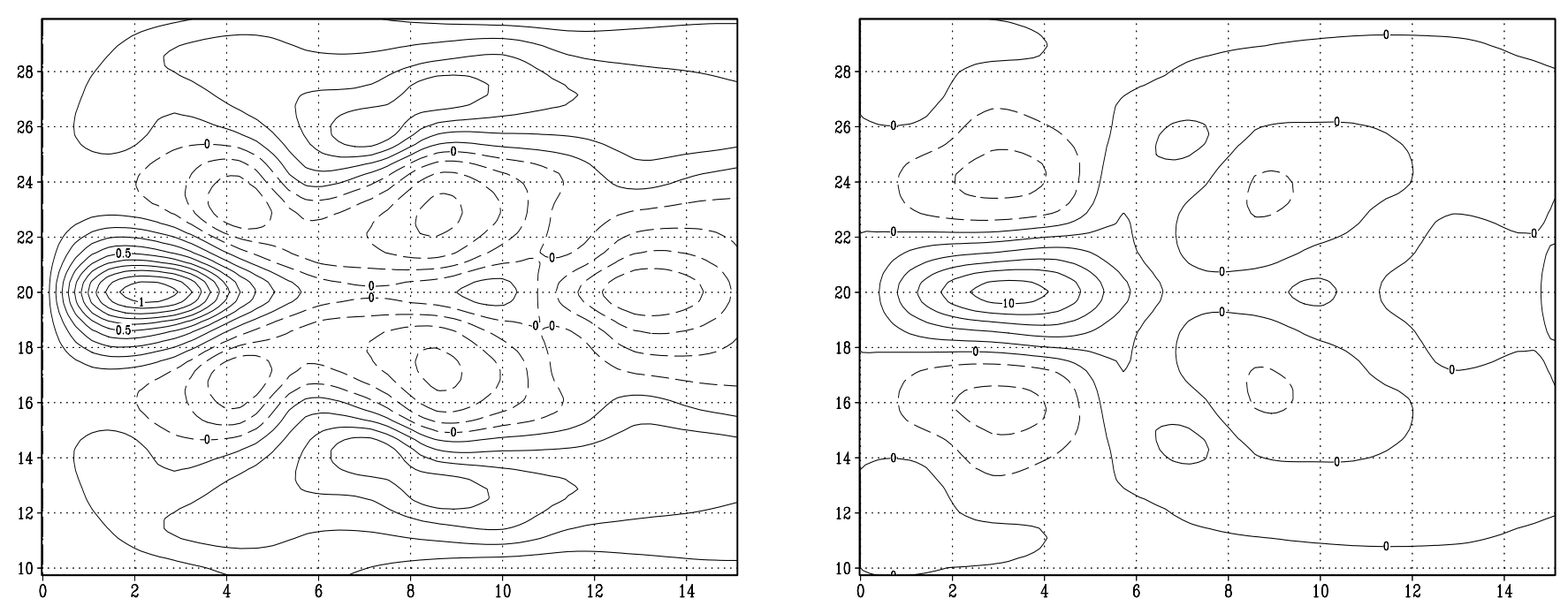

Fig. 11. Perturbation of the streamfunction average approximated by 3 UPOs (contours from -0.4 to 1.1 interval $0.1 \mathrm{~Sv}$ ) (a) and 6 UPOs (contours from -5 to 10 interval $1 \mathrm{~Sv})(\mathbf{b})$.

$7 \mathrm{~b}$, we can see that the pattern is slightly asymmetrical. This asymmetry results in an error which prohibits the improvement of the correlation between the directly calculated perturbation and the reconstructed one which is perfectly symmetrical.

The amplitude of the perturbation is more difficult to approximate. Even with thirty orbits the precision remains at a $6-10 \%$ level. This fact can be explained by the slow convergence of the first singular value of the matrix $H^{(1)}(L)$. As we can see in Fig. 10b, the first singular value varies a lot when we increase the number of UPOs used in the construction of the matrix. When we first use four orbits with low periods, we get a strong underestimate of the first singular value. This error is due to the fact that these four orbits are antisymmetrical, while all other orbits do not possess any symmetry.

The perturbation of the average solution is reconstructed using 3 and 6 UPOs, as shown in Fig. 11. As one can see, the pattern of the perturbation is close to the pattern obtained with the whole set of 30 UPOs (Fig. 6a, but the amplitude is much lower). The maximum value of the streamfunction's perturbation reconstructed with 3 UPOs is equal to 1 Sv, i.e. 22 times lower than the real perturbation. However, the correlation of patterns Fig. 11a and Fig. $6 \mathrm{~b}$ is equal to $81 \%$. When we reconstruct the perturbation with 6 UPOs (Fig. 11b), the correlation becomes $99.2 \%$, but the maximum value remains 2 times lower than the real perturbation. We need to use all 30 orbits in this approximation to obtain the pattern shown in Fig. $6 \mathrm{~b}$ and an error lower than $10 \%$.

\section{Discussion and conclusions}

In this paper we describe a method of estimates of variations of statistical averages of the barotropic ocean model's attractor caused by perturbations of the right-hand side of the model. To realize this approach, one has to find several orbits numerically. Each orbit must be tested from the point of view of possible bifurcations. This implies the analysis of invertibility of the matrix $D_{\xi}$ (22). If this matrix is degenerated or its determinant is too close to zero, the orbit must not be used in the sensitivity analysis. The elimination of one orbit is not very important because we cannot take into account all UPOs of the system due to its infinite number.

After that, one has to observe the spectrum of the matrix $R=\partial S /\left.\partial \omega^{(0)}\right|_{\omega^{(0)}, T, f}(18)$ and determine whether the matrix possesses multiple eigenvalues. In this case, this orbit must also be eliminated from consideration because we cannot estimate variations of its eigenvalues $\delta \mu_{j}$ by the formula (47). For multiple eigenvalues, the scalar product $\left\langle\varphi_{j}, \varphi_{j}^{*}\right\rangle$ may be equal to zero and perturbations $\delta \mu_{j}$ cannot be calculated from (47).

Having constructed matrices $H^{(1)}$ and $H^{(2)}(60)$, (62), one can analyze their invertibility and kernels in order to determine the possibility of finding a forcing which provides the prescribed perturbation of the average solution. If the prescribed perturbation is orthogonal to the kernel of the adjoint matrix, we can solve the system and obtain the forcing perturbation. If we want to find "the most dangerous forcing perturbation", we can analyze singular vectors and singular values of these matrices.

For the barotropic ocean model, we can state that the prediction obtained using the UPO approach corresponds well to the values of $\delta \bar{\omega}$ and $\delta \sigma$ calculated from long model integration. Linear prediction remains valid up to and including rather high values of $\delta f$. About 20 UPOs are sufficient to construct matrices $H$ for this model.

Finally, we shall note several advantages and shortcomings of this method. The principal advantage is the fact that this is an a priori method. It allows us to determine if there exists a forcing perturbation which produces a given perturbation of the average of the model solution or its mean variance. If this 
forcing perturbation exists, one can find it solving the system (59) or (61).

This method also allows us to determine the forcing perturbation, which maximizes the perturbation in the statistical average of the model solution. Another advantage lies in the fact that any UPO belongs to the attractor. This fact ensures that we discuss the attractor perturbation rather than a particular trajectory. This method does not require a long model integration, which is necessary for the direct method to obtain a good accuracy of the statistical averages. In this paper, we obtain the reconstruction of the perturbation of the attractor, which is more precise, in some sense, than the directly calculated value. The reconstructed perturbation is perfectly antisymmetric with respect to the middle line (as it must be), while even the integration for 10000 years is not sufficient to obtain the exact antisymmetry in the directly calculated value.

This method cannot allow us to take into account possible bifurcations leading to the creation of new periodic orbits and stationary points. This is one of the shortcomings of the method, because new UPOs and equilibria may strongly modify the attractor topology and average values. Thus, if any inverse Hopf bifurcation takes place, a stable equilibrium may arise. In this case, the attractor dimension is suddenly reduced to zero and all the attractor's parameters undergo considerable changes. Unfortunately, the available UPO set does not possess any information about orbits that may appear and the method does not predict these changes.

However, this method can automatically take into account some possible changes in the structure of the attractor. Let us suppose one orbit may become stable under some forcing perturbation. In this case, the attractor of the model becomes a one-dimensional regular attractor without chaos. Its average becomes the average of this "stabilized" UPO. Using this method, we will simply obtain this forcing perturbation as the singular vector of $H^{(1)}$. The reason for this is very simple and can be explained by the following reasoning:

- if any orbit in the UPO set is stable, this orbit is weighted with the infinite weight $w$ defined by (9). (A stable orbit has no positive Lyapunov exponents; hence, the denominator of (9) is equal to zero);

- if any unstable orbit can be "stabilized" by some forcing perturbation, the perturbation of the weight $\delta w$ for this orbit will be very large (theoretically infinite) realizing the difference between a finite weight of the unstable original orbit and an infinite weight of the stable perturbed one;

- to realize this large perturbation of the weight $\delta w$, the vector $h$ in (57) must be collinear to $\delta f$ and must have a large norm;

- if we have an orbit for which the norm of $h^{(k)}$ is very large, we can neglect all other orbits and terms in the formula (60). In this case, the matrix $H^{(1)}$ becomes (dropping the index $k$ )

$$
H_{i, j}^{(1)}=(\eta-\bar{\omega})_{i} h_{j}
$$

- in order to look for singular values and vectors of $H^{(1)}$ we first calculate the product

$$
\begin{aligned}
\left(\left(H^{(1)}\right)^{*} \times H^{(1)}\right)_{i, j} & =\sum_{m}\left(H^{(1)}\right)_{m, i}\left(H^{(1)}\right)_{m, j} \\
& =h_{i} h_{j} \sum_{m}(\eta-\bar{\omega})_{m}^{2} \\
& =h_{i} h_{j}\|\eta-\bar{\omega}\|^{2}
\end{aligned}
$$

- this matrix has an eigenvector $h$ with eigenvalue $v=$ $\|\eta-\bar{\omega}\|^{2}\|h\|^{2}$ because

$$
\begin{aligned}
\left(\left(H^{(1)}\right)^{*} H^{(1)}\right) h & =\sum_{j} h_{i} h_{j}\|\eta-\bar{\omega}\|^{2} h_{j} \\
& =\|\eta-\bar{\omega}\|^{2}\|h\|^{2} h
\end{aligned}
$$

The eigenvalue $v$ is large because we assumed the norm of $h$ to be large, and the eigenvector points in the direction of $\delta f$;

- if we multiply the matrix $H^{(1)}$ by any $\delta f$, we obtain the perturbation of the average solution according to (59)

$\delta \bar{\omega}=H^{(1)} \delta f=\langle h, \delta f\rangle(\eta-\bar{\omega})$

hence, the attractor average will move towards the average of the UPO $\eta$, which is going to become stable.

The main shortcoming of this method lies in the necessity of finding several UPOs numerically. For the barotropic ocean model, the search procedure requires the number of operations equivalent to the $10^{4}-10^{5}$ years model integration. Of course, such a long integration is only possible now for a simple model. Its application to more complicated models, such as the eddy-resolving multilayer model and the primitive equations model would require more powerful computers.

\section{Appendix A Calculation of matrices $H^{(1)}$ and $H^{(2)}$}

In order to calculate matrices $H^{(1)}$ and $H^{(2)}$, we need to calculate the following derivatives for each UPO:

- $R=\partial S /\left.\partial \omega^{(0)}\right|_{\omega^{(0)}, T, f}$ defined by (18) which is used to construct matrix $D_{\xi}(21)$ for the Eq. (22);

- $D_{f}=\partial S /\left.\partial f\right|_{\omega^{(0)}, T, f}$ the right-hand side of the Eq. (22);

- $\partial \eta / \partial \omega^{(0)}$ defined by (26) which is used to construct matrix $G_{\xi}(27)$ for the Eq. (28);

- $\partial \eta / \partial f$ defined by (26) which is used to construct matrix $G_{f}$ for the Eq. (28); 
- $\left(\partial R / \partial \omega^{(0)}\right) \varphi$ defined by (49) which is used to calculate vectors $d_{\xi}$ defined by (50);

- $(\partial R / \partial f) \varphi$ defined by (49) which is used to calculate vectors $d_{f}$ defined by (51);

- $\partial R / \partial T$ defined by (49) which is used to calculate the first component of the vector $d \xi$ defined by (50).

We begin with the time discretisation scheme of the tangent linear model, linearized around the UPO $\omega(t)=$ $S\left(\omega^{(0)}, t, f\right)$ for $t \in[0, T], \omega(T)=\omega^{(0)}(73)$. Let us rewrite (73) in the compact matricial form. Let us suppose that UPO $\omega(t)$ is defined by $\mathrm{M}$ points $\omega^{(0)}, \omega^{(1)}, \ldots, \omega^{(M-1)}, \omega^{(M)}$. The final point of the orbit $\omega^{(M)}$ is equal to its initial point $\omega^{(0)}$. We define matrices

$E_{+}(\tau)=\mathcal{M}+\tau \sigma \mathcal{M}+\tau \mu \mathcal{C}$,

$E_{-}(\tau)=\mathcal{M}-\tau \sigma \mathcal{M}-\tau \mu \mathcal{C}$,

$\mathcal{I}\left(\omega^{(n)}\right) \varphi=-\mathcal{J}\left(\Delta^{-1} \omega^{(n)}, \varphi\right)-\mathcal{J}\left(\Delta^{-1} \varphi, \omega^{(n)}+\beta y\right)$

Then, the schemes (73), (74) writes

$$
\begin{aligned}
& \delta \omega^{1 / 2}=\underbrace{-\frac{\tau}{2} E_{+}^{-1}\left(\frac{\tau}{4}\right) \mathcal{I}\left(\omega^{(0)}\right)}_{A\left(\omega^{(0)}\right)} \delta \omega^{0}+\underbrace{E_{+}^{-1}\left(\frac{\tau}{4}\right) E_{-}\left(\frac{\tau}{4}\right)}_{D(\tau / 4)} \delta \omega^{0} \\
& =\underbrace{\left(A\left(\omega^{(0)}\right)+D(\tau / 4)\right)}_{R^{(1 / 2)}} \delta \omega^{0}=R^{(1 / 2)} \delta \omega^{0} \\
& \delta \omega^{1}=\underbrace{-\tau E_{+}^{-1}\left(\frac{\tau}{2}\right) \mathcal{I}\left(\omega^{(1 / 2)}\right)}_{A\left(\omega^{(1 / 2)}\right)} \delta \omega^{1 / 2}+\underbrace{E_{+}^{-1}\left(\frac{\tau}{2}\right) E_{-}\left(\frac{\tau}{2}\right)}_{D(\tau / 2)} \delta \omega^{0} \\
& =\underbrace{\left(A\left(\omega^{(1 / 2)}\right) R^{1 / 2}+D(\tau / 2)\right)}_{R^{(1)}} \delta \omega^{0}=R^{(1)} \delta \omega^{0} \\
& \delta \omega^{2}=\underbrace{-2 \tau E_{+}^{-1}(\tau) \mathcal{I}\left(\omega^{(1)}\right)}_{A\left(\omega^{(1)}\right)} \delta \omega^{1}+\underbrace{E_{+}^{-1}(\tau) E_{-}(\tau)}_{D(\tau)} \delta \omega^{0} \\
& =\underbrace{\left(A\left(\omega^{(1)}\right) R^{1}+D(\tau)\right)}_{R^{(2)}} \delta \omega^{0}=R^{(2)} \delta \omega^{0} \\
& \delta \omega^{n+1}=\underbrace{-2 \tau E_{+}^{-1}(\tau) \mathcal{I}\left(\omega^{(n)}\right)}_{A\left(\omega^{(n)}\right)} \delta \omega^{n}+\underbrace{E_{+}^{-1}(\tau) E_{-}(\tau)}_{D(\tau)} \delta \omega^{n-1} \\
& =\underbrace{\left(A\left(\omega^{(n)}\right) R^{n}+D(\tau) R^{n-1}\right)}_{R^{(n+1)}} \delta \omega^{0} \\
& =R^{(n+1)} \delta \omega^{0} \quad n=2,3, \ldots, M
\end{aligned}
$$

Finally we obtain

$\delta \omega^{M}=R^{M} \delta \omega^{0}$

Thus, the matrix $R^{M}$ obtained at the $M$ th time step is equal to the matrix $R=\partial S /\left.\partial \omega^{(0)}\right|_{\omega^{(0)}, T, f}$.

Let us now consider the derivative $\partial S /\left.\partial f\right|_{\omega^{(0)}, T, f}=D_{f}$. From the time integration scheme (70) of the model equation
(63), we obtain

$$
\begin{aligned}
\omega^{n+1}= & E_{+}^{-1}(\tau) \mathcal{J}\left(\psi^{n}, \omega^{n}+\beta y\right)+E_{+}^{-1}(\tau) E_{-}(\tau) \omega^{n-1} \\
& +E_{+}^{-1}(\tau) \mathcal{M} f
\end{aligned}
$$

Hence,

$$
\begin{aligned}
\frac{\partial \omega^{n+1}}{\partial f}= & E_{+}^{-1}(\tau) \frac{\partial \mathcal{J}\left(\psi^{n}, \omega^{n}+\beta y\right)}{\partial \omega^{n}} \frac{\partial \omega^{n}}{\partial f} \\
& +E_{+}^{-1}(\tau) E_{-}(\tau) \frac{\partial \omega^{n-1}}{\partial f}+E_{+}^{-1}(\tau) \mathcal{M}
\end{aligned}
$$

Using notations (A1), we obtain the evolution of $\delta \omega$ produced by the time-independent perturbation of forcing $\delta \tilde{f}=$ $\mathcal{M} \delta f$

$$
\begin{aligned}
& \delta \omega^{1 / 2}=\frac{\tau}{2} E_{+}^{-1}\left(\frac{\tau}{4}\right) \delta \tilde{f}=D_{f}^{(1 / 2)} \delta \tilde{f} \\
& \delta \omega^{1}=\tau E_{+}^{-1}\left(\frac{\tau}{2}\right) \delta \tilde{f} \underbrace{-\tau E_{+}^{-1}\left(\frac{\tau}{2}\right) \mathcal{I}\left(\omega^{(1 / 2)}\right)}_{A\left(\omega^{(1 / 2)}\right)} \delta \omega^{1 / 2} \\
& =\underbrace{\left(\tau E_{+}^{-1}\left(\frac{\tau}{2}\right)+A\left(\omega^{(1 / 2)}\right) D_{f}^{(1 / 2)}\right)}_{D_{f}^{(1)}} \delta \tilde{f}=D_{f}^{(1)} \delta \tilde{f} \\
& \delta \omega^{2}=2 \tau E_{+}^{-1}(\tau) \delta \tilde{f} \underbrace{-2 \tau E_{+}^{-1}(\tau) \mathcal{I}\left(\omega^{(1)}\right)}_{A\left(\omega^{(1)}\right)} \delta \omega^{1} \\
& =\underbrace{\left(2 \tau E_{+}^{-1}(\tau)+A\left(\omega^{(1)}\right) D_{f}^{(1)}\right)}_{D_{f}^{(2)}} \delta \tilde{f}=D_{f}^{(2)} \delta \tilde{f} \\
& \delta \omega^{(n+1)}=2 \tau E_{+}^{-1}(\tau) \delta \tilde{f} \underbrace{-2 \tau E_{+}^{-1}(\tau) \mathcal{I}\left(\omega^{(n)}\right)}_{A\left(\omega^{(n)}\right)} \delta \omega^{n} \\
& +\underbrace{E_{+}^{-1}(\tau) E_{-}(\tau)}_{D(\tau)} \delta \omega^{n-1} \\
& =\underbrace{\left(2 \tau E_{+}^{-1}(\tau)+A\left(\omega^{(n)}\right) D_{f}^{(n)}+D(\tau) D_{f}^{(n-1)}\right)}_{D_{f}^{(n+1)}} \delta \tilde{f} \\
& =D_{f}^{(n+1)} \delta \tilde{f} \quad n=2,3, \ldots, M
\end{aligned}
$$

Finally, we obtain

$\delta \omega^{M}=D_{f}^{(M)} \delta \tilde{f}=D_{f}^{(M)} \mathcal{M} \delta f$

Thus, the matrix $D_{f}^{M} \mathcal{M}$ obtained at the $M$ th time step is equal to the matrix $D_{f}=\partial S /\left.\partial f\right|_{\omega^{(0)}, T, f}$.

Derivatives $\partial \eta / \partial \omega^{(0)}$ and $\partial \eta / \partial f$ can now easily be calculated from (26) and (A2), (A6):

$$
\begin{aligned}
& \frac{\partial \eta}{\partial \omega^{(0)}}=\frac{1}{M} \sum_{m=1}^{M}\left(\left.\frac{\partial S}{\partial \omega^{(0)}}\right|_{t=m \tau}\right)=\frac{1}{M} \sum_{m=1}^{M} R^{(m)}, \\
& \frac{\partial \eta}{\partial f}=\frac{1}{M} \sum_{m=1}^{M}\left(\left.\frac{\partial S}{\partial f}\right|_{m \tau}\right)=\frac{1}{M} \sum_{m=1}^{M} D_{f}^{(m)} \mathcal{M}
\end{aligned}
$$


Let us now calculate the derivative $\partial R / \partial \omega^{(0)}$. First of all, we have to estimate the derivative $\partial A / \partial \omega$. As defined in (A2),

$$
\begin{aligned}
A_{i, j}\left(\omega^{(n)}\right)=-2 \tau E_{+}^{-1}(\tau) \mathcal{I}\left(\omega^{(n)}\right) & \\
=2 \tau \sum_{m}\left(E_{+}^{-1}\right)_{i, m}(\tau) & {\left[\sum _ { l , p } \mathcal { J } _ { l , m , p } \left(\Delta_{p, j}^{-1}\left(\omega^{(n)}+\beta y\right)_{l}\right.\right.} \\
& \left.-\sum_{l, p} \mathcal{J}_{p, m, j} \Delta_{l, p}^{-1} \omega_{p}^{(n)}\right] \\
=2 \tau \sum_{m}\left(E_{+}^{-1}\right)_{i, m}(\tau)[ & \sum_{l, p}\left(\mathcal{J}_{l, m, p} \Delta_{p, j}^{-1}-\mathcal{J}_{p, m, j} \Delta_{l, p}^{-1}\right) \omega_{l}^{(n)} \\
& \left.+\mathcal{J}_{l, m, p} \Delta_{p, j}^{-1}(\beta y)_{l}\right]
\end{aligned}
$$

where $\Delta^{-1}=\mathcal{M C}^{-1}$ is the matrix of the inverse Laplacian in the finite element's discretisation, and $\mathcal{J}_{i, m, j}=$ $\left\langle\mathcal{J}\left(p_{i}, p_{m}\right), p_{j}\right\rangle$ is the discretisation of the Jacobian. Then, the derivative $\partial A / \partial \omega$ can easily be written

$$
\begin{aligned}
& \frac{\partial A}{\partial \omega}=\frac{\partial A_{i, j}(\omega)}{\partial \omega_{l}} \\
& =2 \tau \sum_{m}\left(E_{+}^{-1}\right)_{i, m}(\tau) \sum_{l, p}\left(\mathcal{J}_{l, m, p} \Delta_{p, j}^{-1}-\mathcal{J}_{p, m, j} \Delta_{l, p}^{-1}\right)
\end{aligned}
$$

We have from (A2)

$$
\begin{aligned}
& R^{(0)}=I \\
& R^{(1 / 2)}=\left(A\left(\omega^{(0)}\right)+D(\tau / 4)\right) \\
& R^{(1)}=\left(A\left(\omega^{(1 / 2)}\right) R^{1 / 2}+D(\tau / 2)\right) \\
& R^{(2)}=\left(A\left(\omega^{(1)}\right) R^{1}+D(\tau)\right) \\
& R^{(n+1)}=\left(A\left(\omega^{(n)}\right) R^{n}+D(\tau) R^{n-1}\right)
\end{aligned}
$$

then

$$
\begin{aligned}
\frac{\partial R^{(1 / 2)}}{\partial \omega^{(0)}} & =\frac{\partial A}{\partial \omega}=\mathcal{X}^{(1 / 2)} \\
\frac{\partial R^{(1)}}{\partial \omega^{(0)}} & =\frac{\partial A}{\partial \omega} \frac{\partial \omega^{(1 / 2)}}{\partial \omega^{(0)}} R^{1 / 2}+A\left(\omega^{(1 / 2)}\right) \frac{\partial R^{(1 / 2)}}{\partial \omega^{(0)}} \\
& =\frac{\partial A}{\partial \omega} R^{1 / 2} R^{1 / 2}+A\left(\omega^{(1 / 2)}\right) \mathcal{X}^{(1 / 2)}=\mathcal{X}^{(1)} \\
\frac{\partial R^{(2)}}{\partial \omega^{(0)}} & =\frac{\partial A}{\partial \omega} \frac{\partial \omega^{(1)}}{\partial \omega^{(0)}} R^{1}+A\left(\omega^{(1)}\right) \frac{\partial R^{(1)}}{\partial \omega^{(0)}} \\
= & \frac{\partial A}{\partial \omega} R^{1} R^{1}+A\left(\omega^{(1)}\right) \mathcal{X}^{(1)}=\mathcal{X}^{(2)} \\
\frac{\partial R^{(n+1)}}{\partial \omega^{(0)}} & =\frac{\partial A}{\partial \omega} \frac{\partial \omega^{(n)}}{\partial \omega^{(0)}} R^{n}+A\left(\omega^{(n)}\right) \frac{\partial R^{(n)}}{\partial \omega^{(0)}}+D(\tau) \frac{\partial R^{(n-1)}}{\partial \omega^{(0)}} \\
= & \frac{\partial A}{\partial \omega} R^{n} R^{n}+A\left(\omega^{(n)}\right) \mathcal{X}^{(n)}+D(\tau) \mathcal{X}^{(n-1)} \\
= & \mathcal{X}^{(n+1)}
\end{aligned}
$$

Finally, we obtain

$$
\mathcal{X}^{(M)}=\frac{\partial R^{(M)}}{\partial \omega^{(0)}}=\frac{\partial R}{\partial \omega^{(0)}}
$$

The derivative $\mathcal{X}^{(M)}=\partial R^{(M)} / \partial \omega^{(0)}=\partial R_{i, m}^{(M)} / \partial \omega_{p}^{(0)}$ is three-dimensional. If we write the equation (A11) in explicit form, we obtain

$$
\begin{aligned}
\frac{\partial R_{i, m}^{(n+1)}}{\partial \omega_{p}^{(0)}}= & \sum_{l} \sum_{j} \frac{\partial A_{i, j}}{\partial \omega_{l}} R_{j, m}^{n} R_{l, p}^{n}+\sum_{j} A_{i, j}\left(\omega^{(n)}\right) \mathcal{X}_{j, m, p}^{(n)} \\
& +\sum_{j} D_{i, j}(\tau) \mathcal{X}_{j, m, p}^{(n-1)}
\end{aligned}
$$

Hence, the procedure (A12) requires $N^{4}$ operations per time step and is impossible to compute for values of $N$ exceeding 100. Fortunately, in this work, we do not need the explicit form of $\partial R^{(M)} / \partial \omega^{(0)}$; we need only its product with the vector $\varphi$. This fact allows us to decrease the number of operations to $N^{3}$ per time step.

Let us denote

$Q_{i, p}^{(n)}=\sum_{m} \mathcal{X}_{i, m, p}^{(n)} \varphi_{m}$

From (A14) we obtain

$$
\begin{aligned}
Q_{i, p}^{(n+1)}= & \sum_{m} \mathcal{X}_{i, m, p}^{(n+1)} \varphi_{m} \\
= & \sum_{m}\left[\sum_{l} \sum_{j} \frac{\partial A_{i, j}}{\partial \omega_{l}} R_{j, m}^{n} R_{l, p}^{n}\right. \\
& \left.+\sum_{j} A_{i, j}\left(\omega^{(n)}\right) \mathcal{X}_{j, m, p}^{(n)}+\sum_{j} D_{i, j}(\tau) \mathcal{X}_{j, m, p}^{(n-1)}\right] \varphi_{m} \\
= & \sum_{l}\left[\sum_{j}(\frac{\partial A_{i, j}}{\partial \omega_{l}} \underbrace{\left(\sum_{m} R_{j, m}^{n} \varphi_{m}\right)}_{v_{j}^{n}}) R_{l, p}^{n}\right] \\
& +\sum_{j} A_{i, j}\left(\omega^{(n)}\right) Q_{j, p}^{(n)}+\sum_{j} D_{i, j}(\tau) Q_{j, p}^{(n-1)} \\
= & \sum_{l}[\underbrace{}_{W_{i, l}^{n}}\left(\frac{\partial A_{i, j}}{\partial \omega_{l}} v_{j}^{n}\right) R_{l, p}^{n}] \\
& +\sum_{j} A_{i, j}\left(\omega^{(n)}\right) Q_{j, p}^{(n)}+\sum_{j} D_{i, j}(\tau) Q_{j, p}^{(n-1)} \\
= & \sum_{l} W_{i, l}^{n} R_{l, p}^{n}+\sum_{j} A_{i, j}\left(\omega^{(n)}\right) Q_{j, p}^{(n)} \\
& +\sum_{j} D_{i, j}(\tau) Q_{j, p}^{(n-1)}
\end{aligned}
$$

This procedure requires approximately $4 N^{3}$ operations per time step. In order to obtain $d_{\xi}(50)$, we calculate the product

$\left(d_{\xi}\right)_{p}=\frac{\sum_{i} Q_{i, p}^{(M)} \varphi_{i}^{*}}{\left\langle\varphi, \varphi^{*}\right\rangle}$

The matrix $\partial R / \partial T$ is calculated by finite differences

$\frac{\partial R}{\partial T}=\frac{R^{(M+1)}-R^{(M-1)}}{2 \tau}$ 
To obtain the first component of $d \xi$, we need just to calculate

$\left(d_{\xi}\right)_{1}=\frac{\left\langle\frac{\partial R}{\partial T} \varphi, \varphi^{*}\right\rangle}{\left\langle\varphi, \varphi^{*}\right\rangle}$

To calculate $d_{f}$ (51) we perform similar calculations. Let us calculate first the derivative $\partial R / \partial f$. We have from (A10) and (A6)

$$
\begin{aligned}
\frac{\partial R^{(1 / 2)}}{\partial f} & =0 \\
\frac{\partial R^{(1)}}{\partial f}= & \frac{\partial A}{\partial \omega} \frac{\partial \omega^{(1 / 2)}}{\partial f} R^{1 / 2}+A\left(\omega^{(1 / 2)}\right) \frac{\partial R^{(1 / 2)}}{\partial f} \\
= & \frac{\partial A}{\partial \omega} D_{f}^{1 / 2} R^{1 / 2}=\mathcal{F}^{(1)} \\
\frac{\partial R^{(2)}}{\partial f}= & \frac{\partial A}{\partial \omega} \frac{\partial \omega^{(1)}}{\partial f} R^{1}+A\left(\omega^{(1)}\right) \frac{\partial R^{(1)}}{\partial f} \\
= & \frac{\partial A}{\partial \omega} D_{f}^{1} R^{1}+A\left(\omega^{(1)}\right) \mathcal{F}^{(1)}=\mathcal{F}^{(2)} \\
\frac{\partial R^{(n+1)}}{\partial f} & =\frac{\partial A}{\partial \omega} \frac{\partial \omega^{(n)}}{\partial f} R^{n}+A\left(\omega^{(n)}\right) \frac{\partial R^{(n)}}{\partial f}+D(\tau) \frac{\partial R^{(n-1)}}{\partial f} \\
= & \frac{\partial A}{\partial \omega} D_{f}^{n} R^{n}+A\left(\omega^{(n)}\right) \mathcal{F}^{(n)}+D(\tau) \mathcal{F}^{(n-1)} \\
= & \mathcal{F}^{(n+1)}(\mathrm{A} 20)
\end{aligned}
$$

Finally, we obtain

$\mathcal{F}^{(M)}=\frac{\partial R^{(M)}}{\partial f}=\frac{\partial R}{\partial f}$

The derivative $\mathcal{F}^{(M)}=\partial R_{i, m}^{(M)} / \partial f_{p}$ is also three-dimensional. The explicit form of the equation (A20) writes

$$
\begin{aligned}
& \frac{\partial R_{i, m}^{(n+1)}}{\partial f_{p}}=\sum_{l} \sum_{j} \frac{\partial A_{i, j}}{\partial \omega_{l}} R_{j, m}^{n}\left(D_{f}\right)_{l, p}^{n} \\
& \quad+\sum_{j} A_{i, j}\left(\omega^{(n)}\right) \mathcal{F}_{j, m, p}^{(n)}+\sum_{j} D_{i, j}(\tau) \mathcal{F}_{j, m, p}^{(n-1)}
\end{aligned}
$$

Hence, the procedure (A21) also requires $N^{4}$ operations per time step. Similarly, if we do not need the explicit form of $\partial R / \partial f$, we write

$$
V_{i, p}^{(n)}=\sum_{m} \mathcal{F}_{i, m, p}^{(n)} \varphi_{m}
$$

From (A14) we obtain

$$
\begin{aligned}
V_{i, p}^{(n+1)}=\sum_{m} & \mathcal{F}_{i, m, p}^{(n+1)} \varphi_{m} \\
= & \sum_{m}\left[\sum_{l} \sum_{j} \frac{\partial A_{i, j}}{\partial \omega_{l}} R_{j, m}^{n}\left(D_{f}\right)_{l, p}^{n}\right. \\
& +\sum_{j} A_{i, j}\left(\omega^{(n)}\right) \mathcal{F}_{j, m, p}^{(n)} \\
& \left.+\sum_{j} D_{i, j}(\tau) \mathcal{F}_{j, m, p}^{(n-1)}\right] \varphi_{m}
\end{aligned}
$$

$$
\begin{gathered}
=\sum_{l} W_{i, l}^{n}\left(D_{f}\right)_{l, p}^{n}+\sum_{j} A_{i, j}\left(\omega^{(n)}\right) V_{j, p}^{(n)} \\
+\sum_{j} D_{i, j}(\tau) V_{j, p}^{(n-1)}
\end{gathered}
$$

where the matrix $W^{n}$ is the same as in (A16).

In order to obtain $d_{f}(51)$, we calculate the product

$\left(d_{f}\right)_{p}=\frac{\sum_{i} V_{i, p}^{(M)} \varphi_{i}^{*}}{\left\langle\varphi, \varphi^{*}\right\rangle}$

All of these derivatives can be calculated in two model runs as follows. The first run is necessary to calculate the matrix $R$ by (A2) and to solve eigenvalue problems

$R \varphi_{k}=\mu_{k} \varphi_{k}$ and $R^{*} \varphi_{k}^{*}=\bar{\mu}_{k} \varphi_{k}^{*}$

For the second run, we define matrices

$R^{0}=I, \quad D_{f}^{0}=0$,

$\left(\frac{\partial \eta}{\partial \omega^{(0)}}\right)^{0}=0, \quad\left(\frac{\partial \eta}{\partial f}\right)^{0}=0$

and according to (A15), (A24), we define for each $\mu_{k}:\left|\mu_{k}\right|>1$

$Q^{(0, k)}=0 \quad V^{(0, k)}=0$

We use the following iterational procedure for any $k=$ $0,1, \ldots, M-1$

$$
\begin{aligned}
& R^{n+1}=\left(A\left(\omega^{(n)}\right) R^{n}+D(\tau) R^{n-1}\right) \\
& D_{f}^{n+1}=\left(2 \tau E_{+}^{-1}(\tau)+A\left(\omega^{(n)}\right) D_{f}^{(n)}+D(\tau) D_{f}^{(n-1)}\right) \\
& \left(\frac{\partial \eta}{\partial \omega^{(0)}}\right)^{n+1}=\left(\frac{\partial \eta}{\partial \omega^{(0)}}\right)^{n}+R^{n} \\
& \left(\frac{\partial \eta}{\partial f}\right)^{n+1}=\left(\frac{\partial \eta}{\partial f}\right)^{n}+D f^{n} \\
& W^{n, k}=\left(\frac{\partial A}{\partial \omega}\left(R^{n} \varphi_{k}\right)\right) \\
& Q^{(n+1, k)}=W^{n, k} R^{n}+A\left(\omega^{(n)}\right) Q^{(n, k)}+D(\tau) Q^{(n-1, k)} \\
& V^{(n+1, k)}=W^{n, k}\left(D_{f}\right)^{n}+A\left(\omega^{(n)}\right) V^{(n, k)}+D(\tau) V^{(n-1, k)}
\end{aligned}
$$

This procedure provides at the $M$ th step

$$
\begin{aligned}
& R^{M}=R=\left.\frac{\partial S}{\partial \omega^{(0)}}\right|_{\omega^{(0)}, T, f}, \quad D_{f}^{M}=\left.\frac{\partial S}{\partial f}\right|_{\omega^{(0)}, T, f}, \\
& \left(\frac{\partial \eta}{\partial \omega^{(0)}}\right)^{M}=\left.\frac{\partial \eta}{\partial \omega^{(0)}}\right|_{\omega^{(0)}, T, f}, \quad\left(\frac{\partial \eta}{\partial f}\right)^{M}=\left.\frac{\partial \eta}{\partial f}\right|_{\omega^{(0)}, T, f}, \\
& Q^{(M, k)}=\left.\frac{\partial R}{\partial \omega^{(0)}}\right|_{\omega^{(0)}, T, f} \varphi_{k}, \quad V^{(M, k)}=\left.\frac{\partial R}{\partial f}\right|_{\omega^{(0)}, T, f} \varphi_{k}
\end{aligned}
$$

Acknowledgements. The author thanks Christine Kazantsev for very helpful discussions. All the contour pictures have been prepared by the Grid Analysis and Display System (GrADS) developed in the Center for Ocean-Land-Atmosphere Interactions, Department of Meteorology, University of Maryland. 


\section{References}

Auerbach, D., Cvitanovic, P., Eckmann, J.-P., Gunaratne, G., and Procaccia., I., Exploring chaotic motions through periodic orbits, Phys. Rev. Lett., 58, 2387, 1987.

Bernadou, M., Modulef: une bibliothèque modulaire d'éléments finis, Inria, 1988.

Christiansen, F., Cvitanovic, P., and Putkaradze, V., Hopf last hope: spatiotemporal chaos in terms of unstable recurrent patterns, Nonlinearity, 10, 55-70, 1997.

Cvitanovic, P., Invariant measurement of strange sets in terms of cycles, Phys. Rev. Lett., 61, 2729, 1988.

Cvitanovic, P., Dynamical averaging in terms of periodic orbits, Physica D, 83, 109, 1995.

Eckhard, B. and Grossman, S., Correlation functions in chaotic systems from periodic orbits., Phys. Rev. E, 50, 4571-4576, 1994.

Eckhard, B. and Ott, G., Periodic orbit analysis of the lorenz attractor, Zeitschrift für Physik B, 93, 259-266, 1994.

Franceschini, V., Giberti, C., and Zheng., Z., Characterisation of the lorenz attractor by unstable periodic orbits, Nonlinearity, 6 , 251-258, 1993.

G.Branstator and Haupt, S., An empirical model of barotropic atmospheric dynamics and its response to tropical forcing, J. Climate, 11, 2645-2667, 1998.

Gritsoun, A., Fluctuation-dissiation relation on attractors of atmospheric models, Russ. J. Numer. Anal. Math. Modelling, 16, 2001.
Gritsoun, A. and Dymnikov, V., Barotropic atmosphere response to small external actions. theory and numerical experiments, Izvestiya, Atmospheric and Oceanic Physics, 35, 511-525, 1999.

Hunt, B. and Ott, E., Optimal periodic orbits of chaotic systems, Phys. Rev. Lett., 76, 2254, 1996a.

Hunt, B. and Ott, E., Optimal periodic orbits of chaotic systems occur at low period, Phys. Rev. E, 54, 328, 1996 b.

Kazantsev, E., Unstable periodic orbits and attractor of the barotropic ocean model, Nonlinear Processes in Geophysics, 5, 193-208, 1998.

Kraichan, R., Classical fluctuation-relaxation theorem, Phys. Rev., 113, 1181-1182, 1959.

Lorenz, E., Deterministic non periodic flow, J. Atmos. Sci., 20, 130-141, 1963.

Sparrow, C., The Lorenz Equations: Bifurcations, Chaos and Strange Attractors, Springer, 1982.

Trevisan, A. and Pancotti, F., Periodic orbits, lyapunov vectors, and singular vectors in the lorenz system, J. Atmos. Sci., 55, 390398, 1998.

Zoldi, S., Unstable periodic orbits analysis of histograms of chaotic time series, Phys. Rev. Lett., 81, 3375, LANL e-print (http://xxx.lanl.gov) chao-dyn/9802005, 1998.

Zoldi, S. and Greenside, H., Spatially localized unstable periodic orbits of a high-dimensional chaotic system, Phys. Rev. E, 57, 2511, LANL e-print (http://xxx.lanl.gov) chao-dyn/9704005, 1998. 